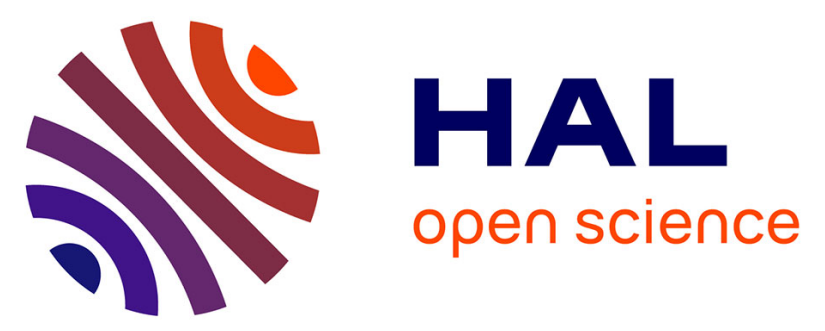

\title{
L'aqueduc de Nîmes dans la haute Vistrenque: analyse interdisciplinaire d'un tronçon souterrain
}

David Carbon, Guilhem Fabre, Philippe Volant, Jean-Luc Fiches, Agnès

Levret, Philippe Combes

\section{- To cite this version:}

David Carbon, Guilhem Fabre, Philippe Volant, Jean-Luc Fiches, Agnès Levret, et al.. L'aqueduc de Nîmes dans la haute Vistrenque: analyse interdisciplinaire d'un tronçon souterrain. Gallia - Archéologie de la France antique, 2005, Aqueducs de la Gaule méditerranéenne, 62, pp.69-86. 10.3406/galia.2005.3221 . hal-01912606

\section{HAL Id: hal-01912606 \\ https://hal.science/hal-01912606}

Submitted on 14 Jan 2020

HAL is a multi-disciplinary open access archive for the deposit and dissemination of scientific research documents, whether they are published or not. The documents may come from teaching and research institutions in France or abroad, or from public or private research centers.
L'archive ouverte pluridisciplinaire HAL, est destinée au dépôt et à la diffusion de documents scientifiques de niveau recherche, publiés ou non, émanant des établissements d'enseignement et de recherche français ou étrangers, des laboratoires publics ou privés.

\section{(ㅇ)(1) $\$$}

Distributed under a Creative Commons Attribution - NonCommercial - NoDerivatives| 4.0 


\title{
L'AQUEDUC DE NÎMES \\ DANS LA HAUTE VISTRENQUE
}

\section{Analyse interdisciplinaire d'un tronçon souterrain}

\author{
David CARBOn, Guilhem FABRE, Philippe VOLANT, \\ Jean-Luc FICHES, Agnès LEVRET et Philippe COMBES
}

\begin{abstract}
Mots-clés. Aqueduc, centuriation, concrétions, déformation, fracturation, hydraulique, pente, photo-interprétation, prospections géophysiques, regards, sismicité.

Résumé. Une exploration et une analyse pluridisciplinaires ont permis de reconnaître, au sud de l'ancien élang de Clausonne (commune de Lédenon), un tronşon souterrain engravé de l'aqueduc de Nîmes, qui se développe sur une longueur continue de près de $500 \mathrm{~m}$. Remarquablement conservé, celui-ci constitue la partie la plus longue de la conduite observée à ce jour dans son parcours souterrain. Après la présentation des contextes géographique et géologique dans lesquels s'inscrit l'aquedur, et en particulier sa partie aval, nous décrivons, pour la première fois, la structure de ce tronçon (géométrie du canal et des regards, matériaux de construction utilisés, pentes), mais surtout nous analysons et interprétons certaines anomalies jusque-là inexpliquées. Une étude des concrétions internes du canal révèle que c'est dans cette zone qu'apparaît leur disposition régressive, qui laisse supposer une perte importante de débit, ou une hydraulique différente. Par ailleurs, d'autres anomalies sont décrites, telle que l'apparition d'une fracturation des encroûtements et des parois du canal. Ces observations permettent d'avancer l'hypothèse que cet ouvrage a pu être affecté, dans la zone où il recoupe la faille de Nîmes, par des déformations d'origine sismique au cours de son fonctionnement ou après.
\end{abstract}

Key-words. Aqueduct, centuriation, concretions, alteration, fractures, hydraulics, slope, photo-interpretation, geophysical surveg;, manholes, seismicit):

Abstract. Through investigations and a pluri-disciplinary analysis, south of the étang de (lausonne (commune de Lédenon), an underground section of the aqueduct of Nimes has been recognized extending continuously on about a length of $500 \mathrm{~m}$. Very well preserved, it presently constitutes the longest part of the aqueduct in its underground course. After an overview of the geographical and geological conlexts, in particular in its downstream part, the structure of this section is for the first time being depicted (geometry of the channel and of the manholes, construction material, slopes) and some unexplained anomalies are analyzed and interpreted. The study of the internal concretions of the conduit shows that their backward disposition appears in this area, which suggests an important loss of the flow or a different hydraulic system. Some anomalies are also described, such as fractures of the encrusting and of channel walls. These observations enable us to put forward the hypothesis that this structure may have been affected, in the area where it intersects the rift of Nimes, by alterations from seismic moves when it was in function or later on.

Translation : Isabelle FALI)LTT

Schlagwörter. Römische Wasserleitung, Zenturiation, Ablagerungen, Verformungen, Riß- und Bruchformationen, Wasserbau, (iefälle, Fotointerpretation, Geophysikalische Prospektion, Revisionsschächte, Seismik.

Zusammenfassung. Südlich des ehemaligen Étang de Clausonne (Gemeinde Lédenon) führle ein pluridisziplinär ausgewverteter Surveg] zur Identifizierung eines auf einer Länge von knapp 500 m unterirdisch verlaufenden, in den Felsen gehauenen Teilstücks der Wasserleitung des römischen Nîmes. Der bemerkenswert gut erhaltene Streckenabschnitt bildet die längste zusammenhängende Partie, die bisher vom unterirdischen Verlauf dieser Leitung bekannt ist. Wir erläutern zunächst den geographischen und geologischen Zusammenhang, in dem die römische Wasserleitung und insbesondere ih. Lnterlauf steht. Arischließend beschreiben wir erstmalig den genauen Aufbau dieses Leitungsabschnitts (Geometrie des Kanals und der Revisionsschächte, verwendetes Baumaterial, (iefälle) und geben vor allem eine Analyse und Interpretation bestimmter Ë̈genheiten, die bisher unerklärt geblieben sind. Eine Untersuchung der Ablagerungen im Inneren des Kanals zeigt, daß diese im fraglichen Abschnitt nur schwach ausgeprägt sind, was auf eine stark reduzierte Schüttung oder sonst eine abweichende Wasserführung schließen läßt. Darïberhinaus werden weitere auffällige Punkle beschrieben, wile etwa Riß-und Bruchformationen des Innenverputzes und der Wände. Diese Beobachtungen führen uns zu der Hypothese, daß das 
Bauwerk in dem Bereich, wo es die geologische Verwerfung von Nimes quert, bereits während seiner Nutzungszeit oder aber im Anschluß daran womöglich von Verformungen seismischen Ursprungs betroffen war:

Übersetzung : Stefan WIRTH

Si l'aqueduc de Nîmes doit sa célébrité au Pont du Gard, la caractéristique principale de cette conduite est d'être le plus souvent enterrée, comme tous les ouvrages romains de grande hydraulique (fig. 67). ('est notamment le cas le long des vingtneuf derniers kilomètres $(58,5 \%$ de la longueur totale du conduit), depuis l'ancien étang de Clausonne jusqu'à Nîmes. Une telle implantation est certes peu favorable pour observer la construction, mais ellc est, en revanche, propice à une bonne conservation du bâti. Les principales contraintes rencontrées sont la difficulté de localiser précisément l'ourrage dans le sous-sol et la présence de la nappe phréatique à faible profondeur qui ennoie presque intégralement l'aqueduc, même en période sèche.

Dans le secteur de la dépression de Clausonne, la portion du canal récemment explorée constitue le plus long tronçon qui ait été reconnu dans la partie souterraine de l'aqueduc. Cela n'aurait pu conduire qu'à des observations relativement banales, tant est monotone le développement de ce conduit, si nous n'avions pas été amenés, par ailleurs, à envisager l'implication de tremblements de terre dans l'histoire du monument pour expliquer des anomalies et des désordres dans la structure de l'ouvrage, et la destruction partielle de l'un des ponts en amont du Pont du (aard (Combes et al., 1997).

Au sortir du domaine des garrigues calcaires, l'aqueduc pénètre dans la plaine de la haute Vistrenque et recoupe le faisceau de la faille de Nîmes dans le secteur de l'ancien étang de Clausonne. C"est dans ce contexte qu'il a été intéressant de rechercher l'origine, jusqu'alors inexpliquée, de la disposition régressive des lamines de croissance du concrétionnement interne de l'aqueduc dans ce secteur, en rapport avec la baisse du niveau de l'eau dans le canal, pouvant être liée à des fuites anormales d'eau durant son fonctionnement. L.hypothèse d'une déformation de l'ouvrage consécutif à un mouvement récent de la faille de Nîmes a été avancée (Fabre, Levret, 2000). Par ailleurs, ces nouvelles observations semblent corroborer l'interprétation en termes de destruction d'origine sismique des désordres étudiés au pont de la lône (Combes el al., 1997).

\section{LE CADRE GÉOMORPHOLOGIQUE DE LA HAUTE VISTRENQUE}

En aval du tumnel qui passe sous le village de Sernhac et jusqu'au-delà de Bezouce ${ }^{57}$, à son retour en garrigue entre

57. Cartes topographiques de l’I(iN: Nîmes 29-42 au $1 / 50000$, Beaucaire-Tarascon et Nimes ouest au 1/25000) carte géologique: Nîmes au 1/50)(00), et notice, BR(;M, 1973, par A. Bonnet, F. Menillet, (3. Berger, I. Coubès ; carte des substances utiles : Nîmes au 1/50 000, BRCM, 1968, B. I emaire dir. ; carte géomorphologique du I anguedoc oriental au 1/200000, 1984 par (;. Fabre.
Saint-Gervasy et Marguerittes, l'aqueduc a été implanté en tranchée, de sorte que son souvenir n'a été conservé qu'en raison de la présence de regards antiques, réutilisés pour puiser de l'eau à l'époque moderne (fig. 68 et 69). Ainsi, depuis la fin de sa gestion antique, il fonctionne en citerne par le drainage de l'aquifère supérieur de la Vistrenque.

Au sud de Sernhac (à partir de la voie ferrée: Fabre $e t$ al. dir., 2000, p. 99-100, n⿳118), l'aqueduc traverse le plateau laniéré du Grès de Clausonne, terminaison orientale des Costières du Rhône gardoises, où passe la limite des bassins versants superficiels du Gardon et du Vistre. Il contourne ensuite par le nord et l'ouest la cuvette de Clausonne, puis, jusqu'au droit de Bezouce, s'inscrit dans une topographie à faibles déclivités qui se distribuent selon deux axes principaux : nord-est - sud-ouest, plat à faiblement pentu (moins de $1 \%$ en moyenne), et nord-sud, un peu plus marqué (autour de $2 \%$ ). On est là à la tête du bassin versant du Vistre, en contrebas de la base du piémont de la garrigue crétacée de Nîmes. I.espace hydrogéomorphologique concerné est exclusivement détritique. Il est modelé depuis la fin du Pléistocène inférieur (Arnal et al., 1974), après le dépôt des formations alluviales distales du Rhône et de la Durance, par une ćrosion aréolaire et linéaire ; celles-ci sont aujourd'hui très contrôlées par les faits anthropiques, particulièrement dynamiques dans ce secteur à substrat meuble.

L'aqueduc s’inscrit successivement dans deux unités géomorphologiques bien distinctes:

- la cuvette endoréique de Clausonne, déjà étudiée dans le détail (Fabre et al., 1999), a été évidée principalement par érosion éolienne, au Pléistocène supérieur et à l'Holocène dans un contexte de microtassements lithodifférenticls, favorisés par la présence d'une nappe phréatique de subsurface ;

- en aval, jusqu'à Bezouce, le canal est implanté dans une topographie générale mixte d'épandages alluviaux siliceux distaux (rhodano-duranciens), largement couverts, vers Bezouce et au-delà, par des dépôts colluviaux de base de glacis d'érosionaccumulation, à matériaux carbonatés proximaux (Fabre, 1984).

Sur les plans pédologique et lithostratigraphique, la tranchée dans laquelle est implanté le canal s'inscrit successivement dans trois unités :

- depuis Le Grès (tranchée SNCF de Sernhac) jusquà la bordure nord de la cuvette de Clausonne, des sols peu épais et peu évolués d'érosion, légèrement brunifiés, au toit du Langhien inféricur marno-gréseux et molasso-gréseux (Fabre, 2000) ;

- de la rive ouest de l'étang de Clausomne au passage de la voie ferrée à Bezouce, des sols profonds fersiallitiques brun rougeâtre, à réserve calcique ou lessivés (avec hydromorphic dans l'ancien étang, moins en aval), au toit des formations fluviatiles allochtones siliceuses du Pléistocène ancien ;

- en aval, des sols carbonatés bruns ou brunifiés, moyeunement et inégalement épais, au toit des formations hétérogènes 


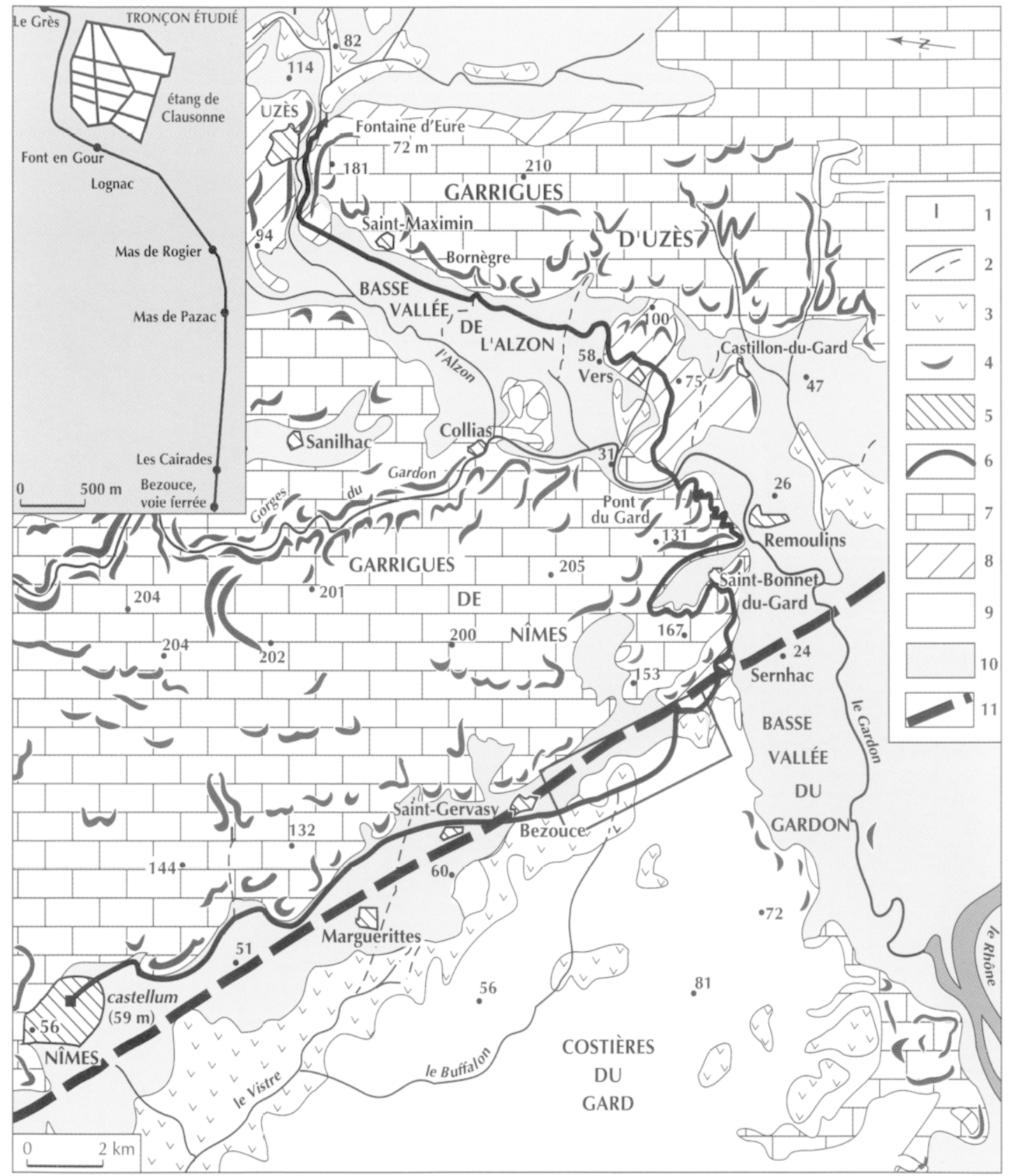

Fig. 67 - L'aqueduc de Nîmes dans son cadre naturel: 1, source; 2, cours d'eau pérenne/temporaire; 3, secteur mal drainé ou inondable, limon gris des anciens marécages; 4, rebord de plateau ou de butte; 5, ville, village; 6, aqueduc antique; 7, plateau karstique des garrigues (Crétacé); 8, butles de calcaire molassique (Miocène); 9, bas plateau caillouteux des Costières; 10, vallée ou dépression à sols profonds; 11 , tracé général de la faille de Nìmes (dessin G. Fabre et J. Vaudour; CNRS; IAO M. Sintès-Aioutz, CNRS).

autochtones carbonatées du Pléistocène moyen-supérieur et de l'Holocène.

À l'époque de la construction de l'aqueduc, le paysage géomorphologique du piémont devait être proche de l'actuel, présentant un profil plus régulier et un peu moins évidé que celui du Néolithique (Chabal, 1997, p. 111-114). Les nombreuses observations effectuées lors de fouilles archéologiques à Nîmes et alentour indiquent que la valeur de l'accrétion sédimentaire pour la période postantique est en movenne de $1 \mathrm{~m}$ à $1,50 \mathrm{~m}$, parfois moins 58 comme on l'a observé, sur l'aqueduc même, à Font en Gour (Fabre, Pey; 1996). Les environnements paléobotanique et paléoécologique

58. Ce chiffre est à rapprocher de l'eustatisme négatif de $1 \mathrm{~m}$ à $1.50 \mathrm{~m}$ de la mer Méditerranée, arancé, pour la période antique (cartes géologiques au 1/50 000 et notices par F. Barer et X. Poul, BRGM-DDA du Gard, 1975 : feuilles, Arles, 1987 ; Les Saintes-Maries-de-la-Mer, 1975: I.e Grau-du-Roi, 1993). 

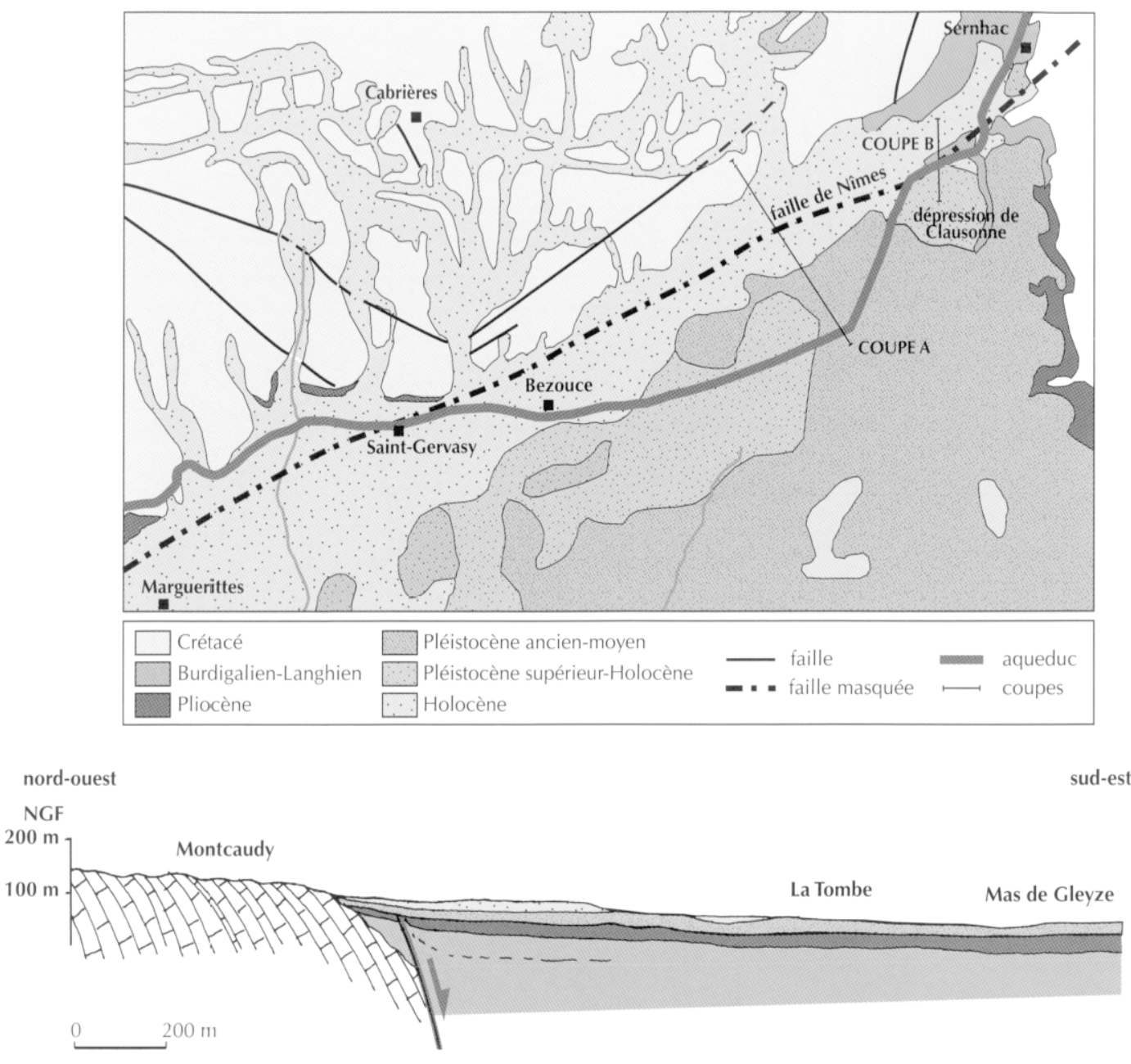

COUPE A

Fig. 68 - Carte el coupe géologiques simplifiées de la haute Vistrenque (dessin G. Fabre, CNRS ; DAO D. Carbon, GEO-TER).

sont inconnus. Il est cependant logique de concevoir, comme en moyenne Vistrenque (sur des sites voisins du Moulin Villard et de la Grande Terre à Caissargues, au sud de Nîmes : Chabal, 1997), la présence, sur la racine du piémont et sur son secteur médian, d'une chênaie verte dominante, laissant place à des vignes, des olivettes et des fruitiers, des cultures céréalières en contrebas, et à une frênaie dégradée en bordure des cours d'eau.

On ne possède aucune donnée sur l'hydrologie de surface et souterraine antique, sauf vers Clausonne où l'étang a été asséché par un canal de drainage souterrain vers le ruisseau de Bournigue au moment de la construction de l'aqueduc 59 .

59. F. Mazauric (1910) estimait que le creusement de ce valat était lié au drainage antique de l'etang. Nous ne saurions souscrire complètement à cette position car le langhien inférieur est bien entaillé sur plusieurs mètres en tête du bassin versant, et l'amplitude du creusement est proche de $10 \mathrm{~m}$ pour quelque $50 \mathrm{~m}$ de largeur des levres au niveau du talus qui délimite la bordure orientale des Costières du Gard dans ce secteur.
Néanmoins, des précisions sur les évolutions hydriques postérieures ont pu être mises en évidence, comme des diffluences hypogées originales à partir du canal vers le Gardon et le Vistre (Fabre et al., 1999). En tout état de cause, le tracé naturel général des petits cours d'eau affluents du Vistre et du ValatNeuf de Lognac-Parac, sis au sud-est de Clausonne, ne devait pas être trop éloigné de l'actuel. Il en va différemment pour la fluctuation de la nappe phréatique. Elle affleurait dans l'étang de Clausonne avant son assèchement antique, alors que son niveau piézométrique moyen actuel se situe à près de $2 \mathrm{~m}$ sous le plancher de l'étang asséché ${ }^{60}$, soit une situation proche de celle du milieu du XIX ${ }^{\circ}$ s. quand C. Dombre notait qu'entre Bezouce et Clausonne, le canal était entièrement noyé, parfois jusqu'à $0,70-0,80 \mathrm{~m}$ au-dessus de l'extrados du canal (Dombre, 1845).

60. Carte hydrogéologique de la Vistrenque au 1/50000 par F. Baver et X. Poul, BRGM-DD $A$ du Gard, 1975. Voir aussi Fabre dans Fabre at al. dir., 2000, p. 159-167. 


\section{LE CONTEXTE STRUCTURAL}

\section{LE FAISCEAU DE FAILLES DE NÎMES}

Sur le plan structural, le tracé de l'aqueduc se situe à l'aplomb de la terminaison nord-est du fossé d'effondrement de la Vistrenque, délimité au nord, par la faille de Nîmes, de direction nord-est - sud-ouest et inclinée à $70^{\circ}$ sud-est en surface, et au sud par une faille parallèle antithétique (Bénédicto, 1996). La faille de Nîmes constitue, avec celles des Cévennes et de la moyenne Durance, l'un des accidents de socle majeur du sud-est de la France. D'une longueur supérieure à $150 \mathrm{~km}$, elle s'étend du golfe du Lion au mont Ventoux. Faille complexe, elle est, en fait, constituée par plusieurs segments bien identifiés d'une longueur moyenne comprise entre 50 et $70 \mathrm{~km}$. Ceux-ci ont contrôlé différemment la géométrie et le style structural de la couverture sédimentaire lors des épisodes extensifs, mésozoïques et oligocènes, et compressifs, pyrénéo-alpins. En effet, le segment central (SèteNîmes) a été très actif durant l'extension oligo-aquitanienne, favorisant un dépôt sédimentaire de 2500 à $4500 \mathrm{~m}$ de puissance dans le fossé d'effondrement de la Vistrenque, alors que le segment nord (Nîmes-mont Ventoux) est resté peu actif durant cette période. En revanche, durant le Plio-Quaternaire, le segment nord présente des indices d'activité tectonique, alors que les autres segments, au sud, apparaissent fossilisés par les dépôts marins pliocènes (Combes, Carbon, 1997). Des marques de ruptures en surface liées à au moins un paléoséisme ont d'ailleurs été mises en évidence dans la région de Courthézon (Vaucluse), à l'aplomb du tracé de la faille de Nîmes. D'après la géométrie de ces ruptures, ce ou ces paléoséismes pourraient avoir atteint une magnitude supérieure à 6 sur l'échelle de Richter. L'âge des dépôts sédimentaires affectés lui confère une datation comprise entre 250000 et 2000 ans (Carbon et al., 1993 ; Combes et al., 1993 ; Granier, Combes, 1997). Néanmoins, malgré ces indices d'activité récente, cette faille ne semble pas avoir rejoué de façon significative à l'Holocène (Peulvast et al., 1999). Ainsi dans le secteur de l'ancien étang de Clausonne, elle est peu marquée dans le relief, depuis le Messinien.

\section{LES DÉFORMATIONS NÉOTECTONIQUES PRÈS DE LA DÉPRESSION DE CLAUSONNE}

Immédiatement au nord de la dépression de Clausonne, en bordure de l'autoroute A9, les sables et cailloutis de l'Astien ont basculé contre la faille de Nîmes avec un pendage de $40^{\circ}$ vers le nord-ouest. Ils participent d'une portion de l'anticlinal généré par une compression pléistocène. Près de Lédenon, une coupe montre une faille inverse faisant chevaucher les calcaires de l'Hauterivien supérieur sur des conglomérats pliocènes et qui semble affecter également les dépôts pléistocènes sus-jacents (fig. 69). Au sud de Sernhac, le Langhien plonge sous le Pliocène en direction du nord-ouest vers la faille de Nîmes. Ce même Pliocène se retrouve perché sur la butte monoclinale dominant Sernhac. Le décrochement vertical post-Langhien induit par l'activité de la faille de Nîmes serait ici d'une centaine de mètres.
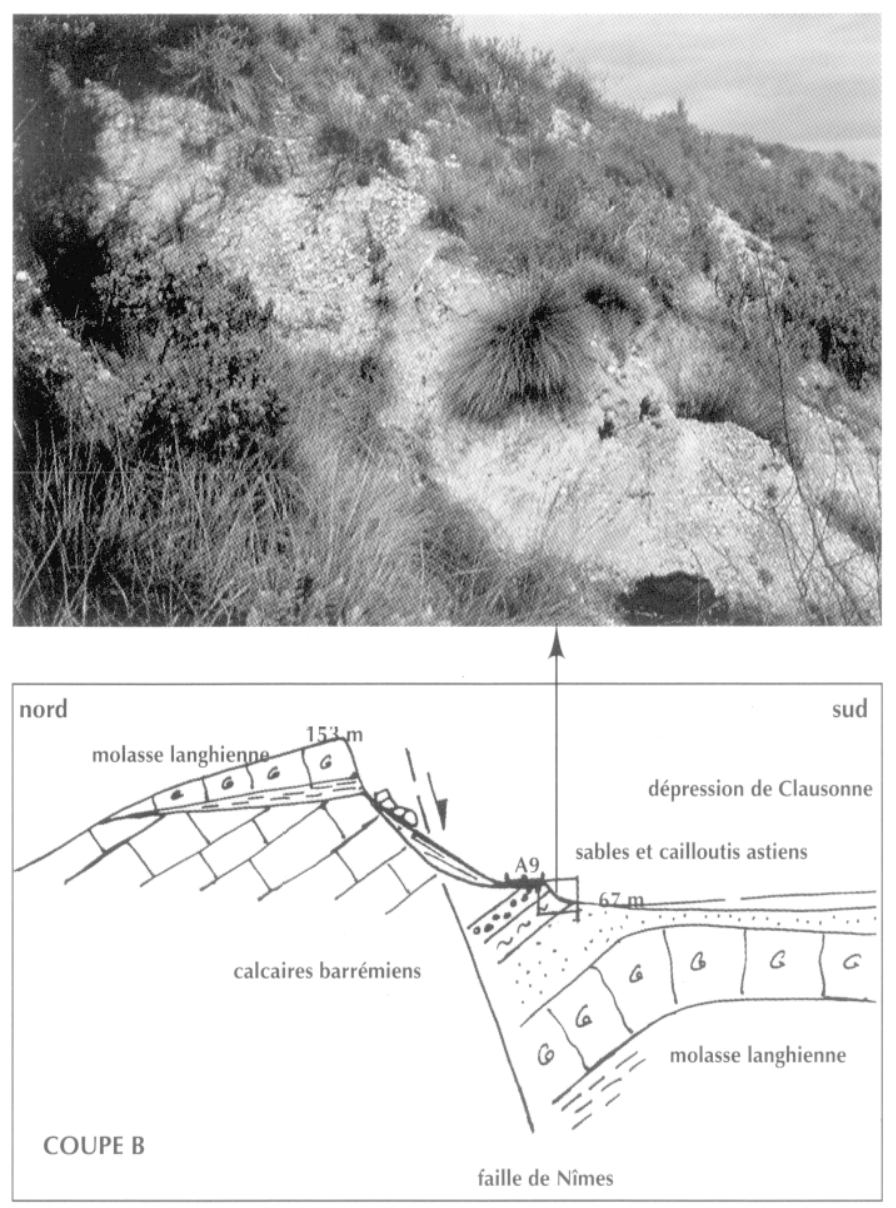

Fig. 69 - Coupe géologique au droit de Clausonne (voir fig. 68) (relevé, cliché et DAO D. Carbon, GEO-TER).

\section{HISTORIQUE DES RECHERCHES ARCHÉOLOGIQUES}

Dans le dernier quart du XVIII ${ }^{\mathrm{e}}$ s. et au XIX ${ }^{\mathrm{e}}$ s., les nombreuses recherches et les travaux effectués pour alimenter en eau la ville de Nîmes (Clary, 2000) ont conduit à s'intéresser de près à la canalisation antique, certains envisageant de réutiliser l'aqueduc, totalement, en partie ou en suivant l'ancien tracé avec un nouveau conduit (Veyrac, 1999 ; Clary et al., 2000). I.e témoignage le plus précis est celui de Charles Dombre qui procéda, en 1844-1845, à des dégagements et des explorations entre le Pont du Gard et Nîmes. Cette entreprise donna lieu à un dossier cartographique, malheureusement conservé aujourd'hui de façon très lacunaire, et au levé de 460 profils situés à une distance moyenne de $70 \mathrm{~m}$ (Dombre, 1845). Cette étude, largement utilisée par la suite, non sans erreurs parfois (Espérandieu, 1926; Grenier, 1960), représente la première base scientifique de nos connaissances sur le tracé général du canal et son anatomie, jusqu'aux reconnaissances systématiques, effectuées sur le terrain dans les années $1980^{61}$.

61. Ces recherches pluridisciplinaircs, qui se poursuivent cncore, ont été initiées sous la direction de G. Fabre et de J.-L. Fiches dans le cadre 
Dans cette zone qu'il appelait « la plaine de Paza », Dombre avait reconnu l'une des trois parties de belle longueur $(8747 \mathrm{~m}$ au total) où la voûte était conservée; une autre se trouve encore aux abords de Sernhac où l'on a pu l'observer à partir de cinq regards utilisés comme puits dans le village (Fabre $e t$ al. dir., 2000, p. 99, n⿳117); une troisième existait à l'ouest de Saint-Gervasy, mais cette dernière a été détruite lors de la construction, au XIX ${ }^{\mathrm{e}}$ s., du canal dit du Pouzin qui a repris la majeure partie de l'ouvrage antique entre Bezouce et les abords de Nîmes (Clary, 2000) et seul un plan de Dombre rend compte des fouilles dont elle fit l'objet en 1844 (Fabre t al. dir., 2000 , p. 103).

Lorsque les recherches ont repris dans les années 1980, on n'a pas pu faire d'observations précises sur $5 \mathrm{~km}$ entre un point, Le Grès, où une tranchée pour le chemin de fer avait coupé le canal au nord de l'étang de Clausonne (Fabre et al. dir., 2000, p. 99-100, $\mathrm{n}^{\circ} 118$ ) et le point où la voie ferrée repasse au-dessus du canal à Bezouce (ibid., p. 102, $\mathrm{n}^{\circ} 125$ ). Or, ce tronçon présentait un intérêt majeur en raison de l'évolution de la géométrie du concrétionnement interne du conduit qui était observée à ses extrémités (fig. 67). En effet, les dépôts carbonatés, qui ont une disposition stratigraphique transgressive au point 118 comme partout à l'amont, montrent une disposition régressive des dernières lamines claires et des dépôts terrigènes les plus récents au point 125 , ce qui implique, dans l'intervalle, un net abaissement du niveau de l'eau dans le canal au cours de son fonctionnement. Ce tronçon est donc l'un de ceux qui ont fait l'objet des travaux les plus récents et encore inédits (présentation très partielle dans Fabre et al. dir., 2000, p. 101-102, n"s 119, 119A et 121).

En août-septembre 1995 , le curage à la pelle mécanique d'un fossé de drainage en bordure ouest de l'étang de Clausonne, au lieu-dit La Source de Font en Gour, a mis au jour un important tronçon de l'aqueduc situé à l'aval d'un ancien puits à roue implanté à l'emplacement d'un regard. Dans le cadre d'une étude pluridisciplinaire "Archéosismicité " (19952003) orchestrée par l'Institut de radioprotection et de sûreté nucléaire (IRSN), des investigations de terrain ont été réalisées dans ce secteur, de manière à analyser l'évolution de la géométrie des concrétionnements carbonatés dans le canal et les éventuelles déformations de sa structure (fissures, décalages...) susceptibles d'être en relation avec l'abaissement significatif du niveau de l'eau dans le canal.

En octobre 1998, des mesures de nivellement ont été effectuées à Font en ('our et au Mas de Pazac à l'aide d'un récepteur GPS et d'un théodolite. Rattachées au réseau nivelé de l'Institut géographique national (IGN) par l'intermédiaire d'une seconde antenne GPS implantée sur un repère de nivellement de l'IGN, elles avaient pour but de reconstituer le profil en long du canal entre l'étang asséché de Clausonne et le regard de la voie ferréc à Bezouce, afin de détecter d'éventuelles anomalies dans la pente.

de LATP du (NRS, Archéologie métropolitaine, projet: "Laqueduc romain de Nîmes et le Pont du Gard, archéologie, géosvstème et histoire " (1984-1989) et de fouilles programmées conduites par J.-L. Fiches et J.-L.. Paillet; voir l'historique des recherches dans Fahre, Fiches, 2001.
Le 2 novembre 1998, une exploration du puits du Mas de Pazac a été réalisée en plongée, avec des prises de vue photographiques et des mesures du dimensionnement du canal.

En juin 1999, suite à un étiage exceptionnel, l'aqueduc a été exploré à partir des fouilles de Font en Gour. Afin de pouvoir positionner en plan l'axe du tracé sur la longueur du tronçon prospecté, un pentadécamètre a été utilisé pour mesurer la position des observations à partir du point d'origine (situé à l'entrée du canal à Font en Gour). L'azimut de l'axe de l'aqueduc a été reporté tous les $50 \mathrm{~m}$ (fig. 70). Ainsi, le canal a été repéré sur $485 \mathrm{~m}$ jusqu'à une trémie qui correspond vraisemblablement à un effondrement ponctuel de la voûte avec décharge des matériaux de remplissage de la tranchée susjacente (formation détritique du Villafranchien). Un premier effondrement partiel de la voûte avait été rencontré à $284 \mathrm{~m}$ de l'entrée.

En mars, puis en octobre 2000, une exploration de l'aqueduc a été réalisée à partir d'un puits-regard situé dans le bâtiment nord-est du Mas de Gleyze ou Mas de Rogier, sur une distance de $40 \mathrm{~m}$ en amont, jusqu'à une trémie d'effondrement, alors qu'en aval, le canal est comblé jusqu'à la voûte sur au moins $10 \mathrm{~m}$ de longueur.

En octobre 2000, les mesures de nivellement de l'aqueduc à Sernhac (Le Grès) et Bezouce (voie ferrée) ont été reprises et complétées. En définitive, l'aqueduc a été nivelé en chaque point d'observation accessible à ce jour dans ce secteur (Le Grès, Font en Gour, Mas de Gleyze, Mas de Pazac et Bezouce, voie ferrée).

\section{LA LOCALISATION DE L'AQUEDUC}

Entre Font en Gour et le Mas de Pazac, le tracé de l'aqueduc n'était connu qu'approximativement. Quelques zones d'effondrement dans les champs et quelques débris maçonnés retrouvés dans les fossés marquaient ponctuellement la position de l'ouvrage antique.

Pour préciser celle-ci, mais aussi celle du canal du Pouzin qui le longe, les informations apportées par les photographies aériennes, les documents cartographiques anciens et les résultats d'investigations géophysiques ont été confrontés. I a synthèse de ces données permet de proposer un tracé fiable de l'aqueduc.

\section{LE TRACÉ SUR LES PIOTOGRAPIIIES AÉRIENNES ET LES CARTES ANCIENNES}

Le positionnement des canaux souterrains a été réalisé à partir de l'analyse de photographies aériennes prises à différentes époques ${ }^{62}$. En effet, les structures archéologiques

62. Ce sont des missions ICN sélectionnées à la photothèque de l'ICiN à Saint-Mandé (noir et blanc, 1942, 1953, 1960), 1965, 1978; couleurs, 1996 ) et de l'Office national des forets (infrarouge, 1990) ainsi que des rues obliques, réalisées à faible altitude (probablement en 1960) à la suite de fortes inondations. Ces dernières (noir et blanc) ont été prises par (3. Durville, géomètre expert à Nîmes, à la demande de la société Vilmorin, propriétaire du domaine du Mas de Parac: ; clles nous ontété gracieusement fournies en 1999 par M. Herlem, de cette société. 
Illustration non autorisée à la diffusion

Fig. 70 - Tracés de l'aqueduc antique el du canal du Pouzin (XIX's.)

entre Font en Gour et le Mas de Pazac sur photographie ciérienne : exemple de la mission I(F,i, 1953 (montage (GEO)TER). 
enterrées à des profondeurs de quelques mètres, ainsi que les éventuelles structures tectoniques n'apparaissent pas de la même façon en fonction de la saison (humidité du sol, réflectivité, degré de maturité des végétaux...) et du type de végétation ou de la nature des cultures. L'utilisation de cartes anciennes et de plans cadastraux ${ }^{63}$ a permis également de préciser la position de l'aqueduc du Pouzin et des différentes canalisations d'adduction d'eau de la compagnie du BasRhône-Languedoc qui se trouvent dans ce secteur.

Grâce à la superposition des plans cadastraux avec les photographies aériennes, on a mis en relation la trace des différents linéaments observés avec les limites parcellaires actuelles ou avec des limites disparues à l'occasion de remembrements récents ${ }^{64}$. Avec la mise à la même échelle de tous ces documents, le positionnement du tronçon du canal antique exploré en aval de Font en Gour a été précisé et l'ensemble des traces et anomalies repérées sur les différentes photographies aériennes a été reporté dans un document de synthèse au $1 / 10000$ qui fait apparaître la position de l'aqueduc antique et celle de l'aqueduc du Pouzin relativement parallèles l'un à l'autre (fig. 70). Le long de l'aqueduc romain, d'autres anomalies, linéaments et taches dans les parcelles cultivées ont également été relevés. Certains linéaments, en effet, ne correspondent vraisemblablement pas à d'anciennes limites parcellaires : l'un d'eux, de direction nord-est - sud-ouest (parallèle à la zone de failles de Nîmes), est très nettement visible sur la photographie de 1953, à quelques centaines de mètres au sud de Font en Gour ; un autre, nord-ouest - sud-est, apparaît sur les photographies récentes en couleur et en infrarouge; un troisième, de direction est-sud-est - ouest-nord-ouest, ressort entre la route départementale D500 et le Mas de Gleyze, sur presque toutes les photographies. Ces linéaments ne semblent pas être d'origine anthropique et peuvent correspondre à des contacts lithologiques entre formations différentes ou à des indices morphologiques de la présence de failles en profondeur.

D'autres anomalies observées systématiquement sur les photographies aériennes correspondent à des " taches claires" qui s'alignent le long du tracé de l'aqueduc entre Font en Gour et le Mas de Gleyze, dans les dépôts alluviaux superficiels. Elles pourraient signaler des zones où se seraient formés des encroûtements carbonatés au sein du matériel alluvial, vraisemblablement en relation avec des pertes d'eau le long du canal antique.

\section{INVESTIGATIONS GÉOPHYSIQUES SUR LE TRACÉ DE L'AQUEDUC}

Des investigations géophysiques ont été réalisées le long du tracé de l'aqueduc identifié sur les photographies aériennes de

63. Les cartes topographiques analysées sont les plans au $1 / 20000 \mathrm{de}$ 1779 (levés 1774-1775). d'Arignon au 1/80 000 de 1887, d'Avignon au $1 / 40000$ de 1857 , d'Avignon au 1/50) 000 de 1906 révisćc (levés 1866), de Nîmes n" 3 au 1/20 000 de 1948 et de Nîmes nos 3-4 au 1/25000 de 1974 (levés 1945-1946).

64. Sur la photographie de 1965, un linéament très net de direction estnord-est - ouest-sud-ouest apparaît à l'ouest des bâtisses, Les Mugues. Il se superpose, en fait, à la bordure d'une parcelle visible sur la photographic de 1953. manière à contrôler sa présence en profondeur. Plusieurs méthodes ont été testées, et celle du géoradar s'est avérée la plus satisfaisante, en prenant en compte notamment les paramètres du coût, de la souplesse, de la rapidité et de la pertinence des résultats. Sept profils ont été réalisés transversalement à la structure, entre le Mas de Pazac et Font en Gour.

Dans un premier temps, ils ont été effectués là où le tracé et la profondeur de l'aqueduc étaient connus, de manière à paramétrer correctement le radar en fonction de la nature du soussol, de la hauteur de la nappe phréatique et de la profondeur de l'ouvrage. Ces profils ont permis de visualiser l'image radar donnée par l'aqueduc en profondeur.

En tenant compte des faciès alluvionnaires des premiers mètres, deux radars de conceptions différentes ont été utilisés : - un GSSI américain qui gomme les phénomènes d'échos aériens grâce au blindage de ses antennes,

- un RAMAC suédois, aux antennes non blindées, long à mettre en œuvre, mais plus performant dans les basses fréquences.

Deux fréquences différentes ont par ailleurs été utilisées. En effet, pour des basses fréquences, les anomalies liées à la présence de l'aqueduc ont une forme hyperbolique. La géométrie de la structure et sa profondeur lui confèrent une nature d'objet ponctuel vis-à-vis de ces grandes longueurs d'ondes. Pour les plus hautes fréquences, l'image obtenue est plus détaillée (les murs bajoyers du canal s'individualisent). Dans ce cas, les dimensions de l'aqueduc représentent plusieurs fois la longueur d'onde du signal incident, ce qui donne naissance à un signal réfléchi présentant un niveau de détails auquel la basse fréquence ne permet pas d'accéder. En revanche, la pénétration en profondeur est moindre que pour des longueurs d'ondes plus élevées.

Les résultats de ces investigations ont permis de localiser avec précision la position et la profondeur de l'aqueduc en plusieurs points de son tracé.

\section{ANOMALIE DANS LE TRACÉ EN AVAL DE FONT EN GOUR}

L'exploration du canal antique a permis de déceler une anomalie dans le tracé à $459 \mathrm{~m}$ de l'entrée de Font en Gour (fig. 70, anomalie de structure sud). Celle-ci se caractérise par un décrochement dans le sens senestre de l'axe de l'aqueduc d'une amplitude de 1,20 m environ (soit la largeur du cuvelage). Alors qu'en amont de cette zone, le conduit a une direction rectiligne $\mathrm{Nm} 194^{\circ}$, il décrit un brusque coude vers la gauche pour prendre une direction $\mathrm{Nm} 188^{\circ}$ sur $7 \mathrm{~m}$ arant un nouveau coude vers la droite pour retrouver, vers l'aval, une direction rectiligne $\mathrm{Nm} 194^{\circ}$ (fig. 71). Cette anomalie précède une modification nette de l'orientation de l'aqueduc, on ne peut donc penser, dans cette partie plane et dégagée, qui a, de plus, nécessité le creusement préalable de profondes tranchées, à une rectification nécessaire au point de rencontre de deux équipes qui n'auraient pas suivi exactement le même axe. On verra plus loin qu'une déformation d'origine sismique n'est pas à exclure, mais, si cette anomalie s'inscrit bien dans la conception générale du tracé, on peut avancer l'hypothèse qu'on a tenu compte à cet endroit d'un chemin rural préexistant, recoupé au plus court. La présence d'un chemin 


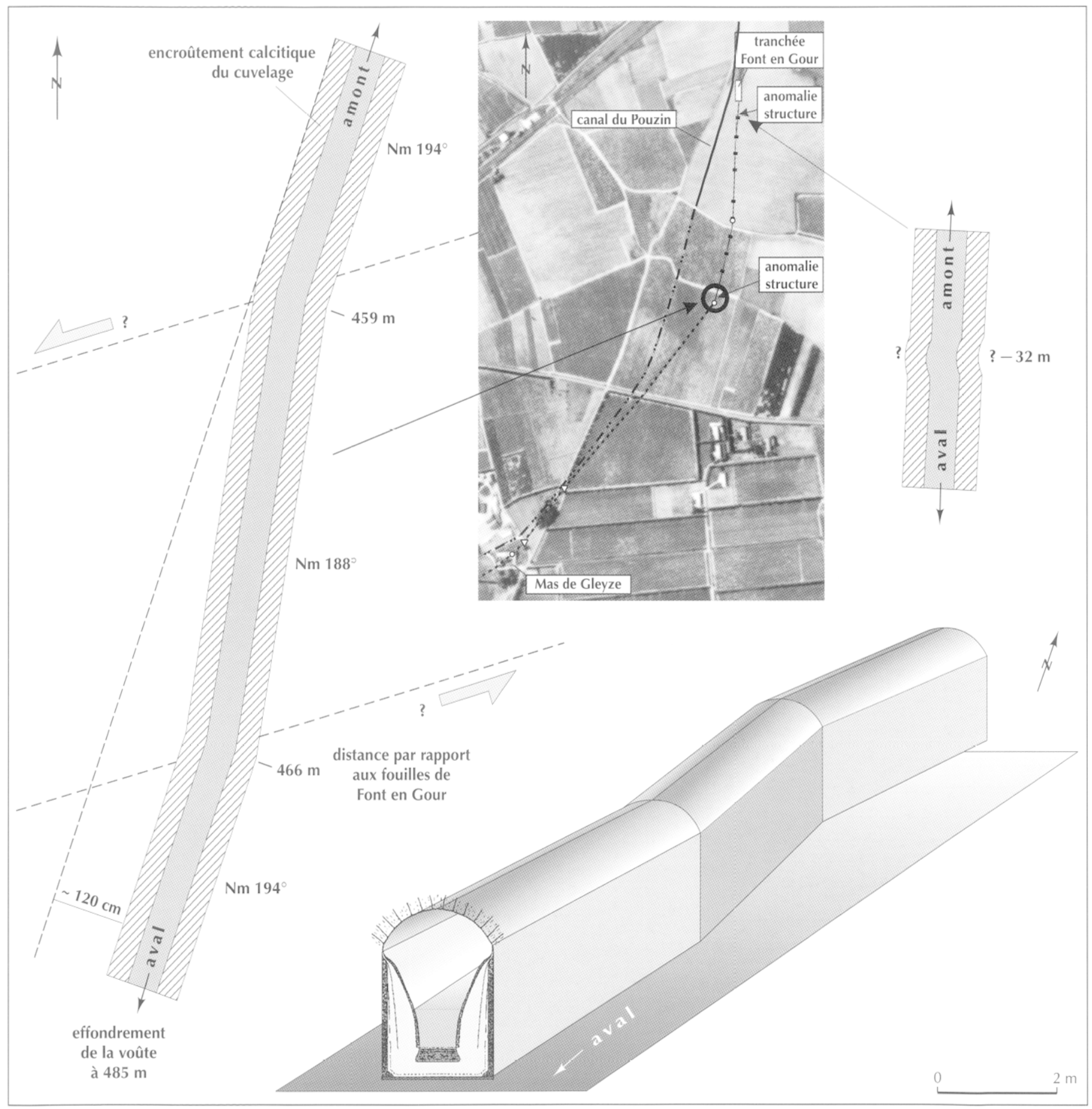

Fig. 71 - Situation, vues en plan et en perspective d'un tronson de l'aqueduc qui présente un décalage horizontal de son axe (1,20 m environ) suivant un mouvement senestre (DAO D). Carbon, (EEO-TER).

rural en ce point est d'ailleurs tout à fait possible. D'une part, si l'on trace de ce point et à la perpendiculaire de l'aqueduc une ligne droite vers l'est jusqu'au bord de la Costière, on aboutit à la tête d'un vallon qu'occupe aujourd'hui le château de Clausonne près duquel se trourait une importante villa équipée d'un atelier d'amphores vinaires (Provost el al., 1999, p. 450). D'autrc part, si l'on sc réfère à l'analsse des formes du paysage local (González. Villaescusa, 2002, p. 148) et à la restitution proposée pour le cadastre A qui était affiché à Orange et qui a pu être mis en place dans la seconde moitié du $\mathrm{I}^{\mathrm{er}}$ s. av. J.-C. (Chouquer, 1983, p. 284-288), on observe qu'un decumanus de cette centuriation (14c limes à droite du decumanus maximus) pouvait passer en ce point ${ }^{65}$, près d'une zone où le

65. On notera que l'orientation des kardines de ce cadastre ( $\left.\mathrm{Xy} 2^{\circ} 30^{\circ}\right)$ est voisine de celle que suit l'aqueduc à cet endroit $\left(\operatorname{Nm} 8^{\circ}=\operatorname{Ng} 6^{\circ}\right)$. 
parcellaire actuel a conservé le souvenir de cette division des terres (fig. 72).

\section{LE CANAL, SES REGARDS, SES PENTES}

Le canal antique ne présente pas de particularité architecturale dans cette zone. Son mode de construction est tout à fait conforme à ce que l'on connaît généralement par ailleurs. Ainsi les murs latéraux sont habituellement distants de $1,33 \mathrm{~m}$ à $1,3 \check{\mathrm{j}} \mathrm{m}$, soit 4,5 pieds romains, et sont couverts d'un béton de tuileau d'une épaisseur moyenne de $5 \mathrm{~cm}$, ce qui réduit la largeur du canal à 1,23-1,25 m (Paillet, 2000, p. 361-362). Les mesures effectuées au Mas de Pazac et à Bezouce (voie ferrée) répondent à la règle générale (fig. 73) : les largeurs moindres, mesurées à Sernhac et à Font en Gour ont été prises au-dessus des encroûtements qui dépassent le cuvelage, en des points où la corde de la voûte est plus réduite. En revanche, au-dessous du regard du Mas de Gleyze, on doit noter que les largeurs du cuvelage et du canal sont légèrement supérieures à la movenne $(1,30 \mathrm{~m}$ et $1,40 \mathrm{~m}$ ).

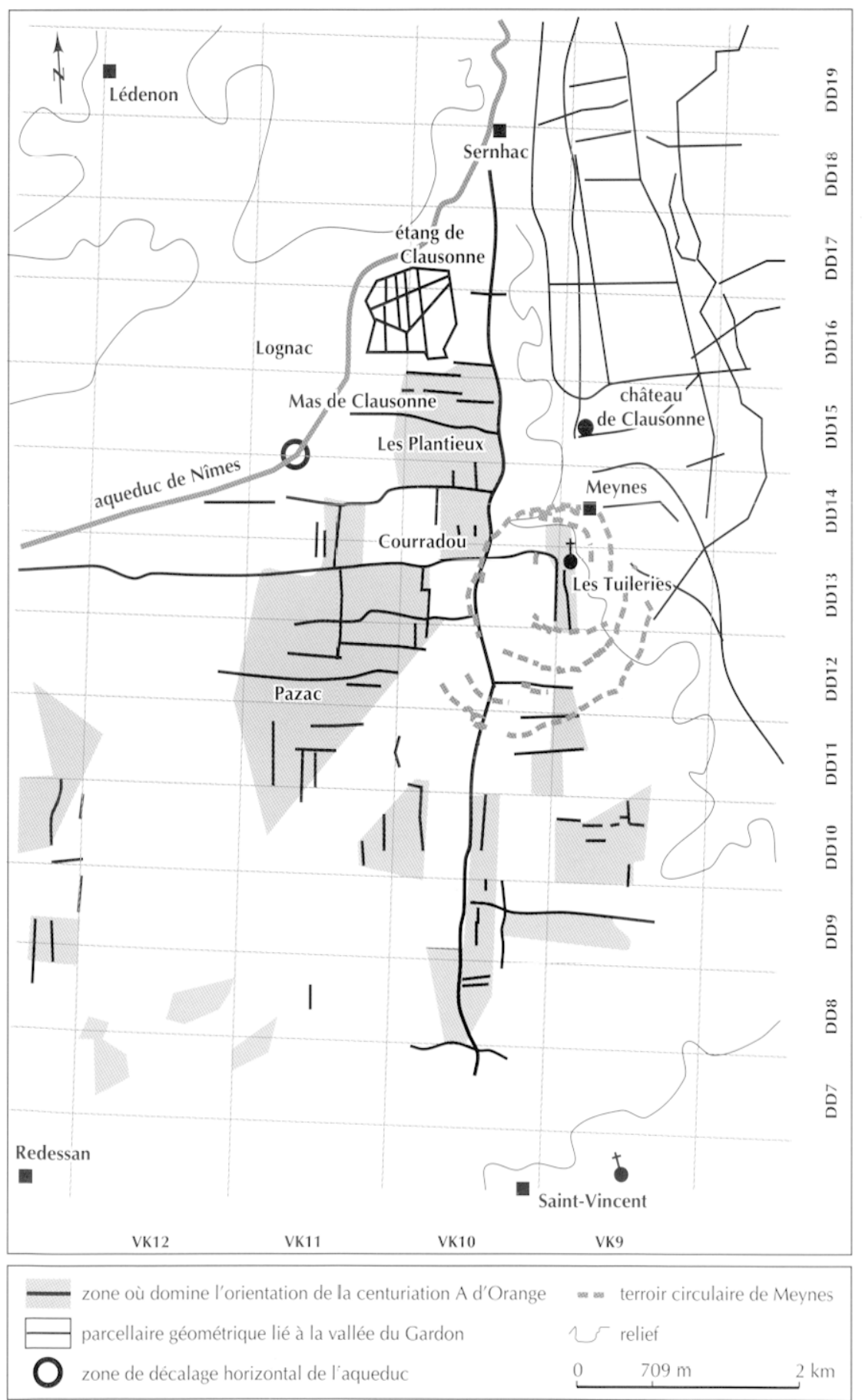

Fig. 72 - Hypothèse de restitulion du cadastre A affiché à Orange ( $\left.\mathrm{Ng} 2^{\circ} 30^{\prime}\right)$ dans la région de l'étang de Clausonne (d'après (ionzález Villaescusa, 2002, p. 170, fig. 29). 


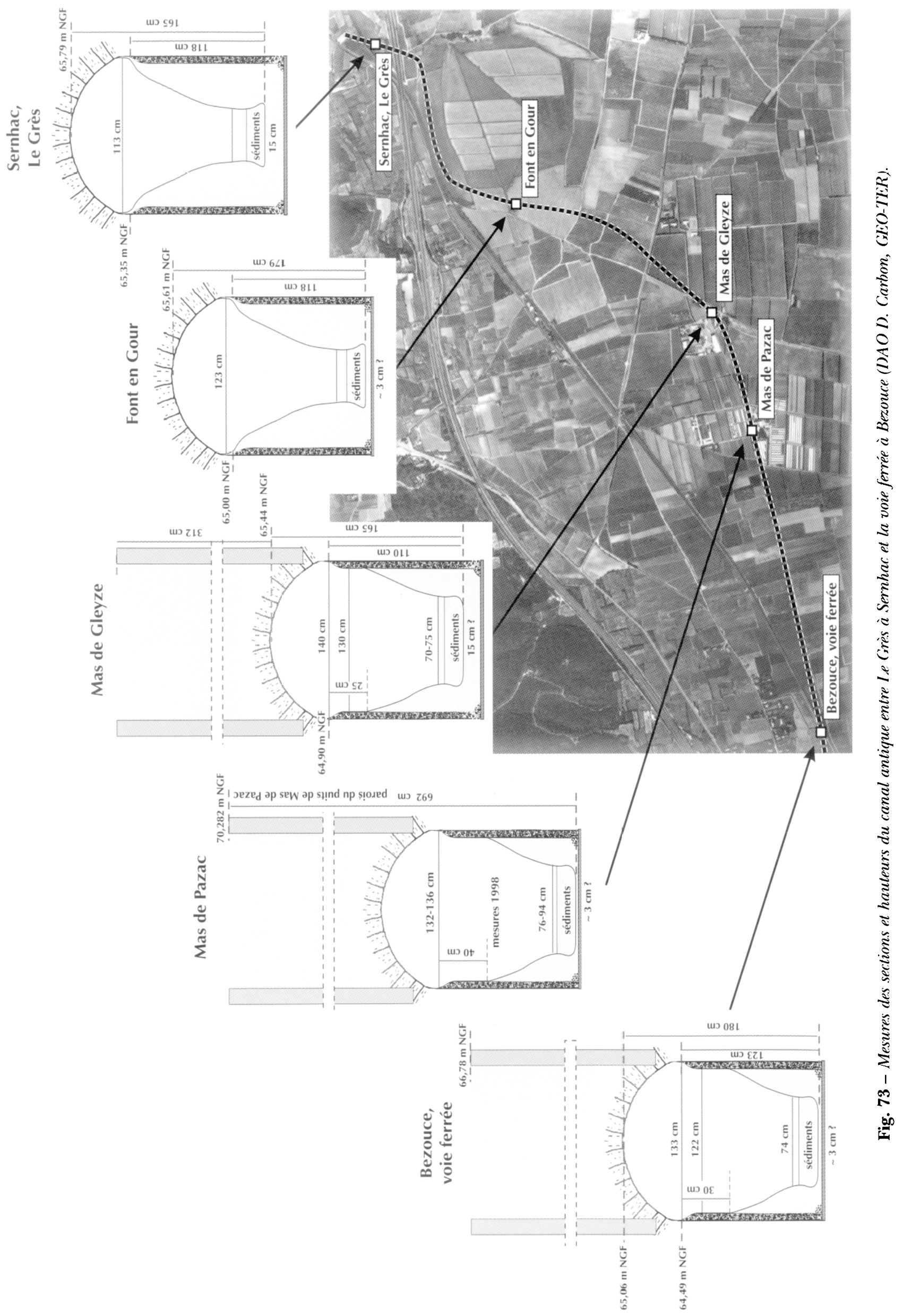


En aval de Font en Gour, on a également observé un changement dans la nature du matériau utilisé, au moins pour les mocllons des regards et parfois les claveaux des arcs situés audessous. En effet, dans la partie amont, la molasse calcarogréscuse langhienne et burdigalienne issue des carrières de Sernhac est utilisée (Fabre, 2000), alors qu'en aval, il s'agit de calcaires gris-beige compacts à pâte fine du HauterivienBarrémien, qui se rencontrent à partir du regard $\mathrm{n}^{\circ} 5$, situé à $239 \mathrm{~m}$ de Font en Gour, et que l'on trouve jusqu'à Nîmes. Leur gisement le plus proche est celui de la bordure méridionale de la garrigue de Nîmes où de nombreuses carrières présentent, sur leurs fronts de taille, des bancs épais de 10 à $40 \mathrm{~cm}$, soit le calibre habituel des pierres utilisées dans la construction du canal avec de faibles retouches. Mais l'intérêt majeur de cette partie de l'aqueduc est d'avoir conservé un nombre important de regards.

\section{LES REGARDS}

En effet, entre Font en Gour et le Mas de Pazac, sur une longueur de $1946 \mathrm{~m}$, on connaît désormais treize de ces puits de visite : dix ont été vus de l'intérieur du canal (fig. 74) et trois autres, à Font en Gour, au Mas de Gleyze et au Mas de Pazac, ont été réutilisés comme puits. Rappelons que sur le reste de l'ouvrage, neuf regards seulement ont été conservés ${ }^{66}$ alors que treize autres se signalent par l'aménagement caractéristique de la voûte ${ }^{67}$, par un effondrement ${ }^{68}$ ou à la transition entre une partie voûtée et une couverture dallée ${ }^{69}$.

Dombre avait observé que les regards, parementés, étaient de section rectangulaire mais de largeur variable $(0,60 \mathrm{~m}$ à $1 \mathrm{~m}$ ), leur plus grande dimension correspondant à la largeur uniforme du canal $(1,30 \mathrm{~m})$. Il avait aussi noté que leur espacement variait de $80 \mathrm{~m}$ à $100 \mathrm{~m}$ dans les segments droits, se réduisant jusqu'à $14 \mathrm{~m}$ dans les coudes. On peut désormais préciser, voire corriger ces informations. En effet, les dix regards successifs qui sont situés à l'aval de Font en Gour sont plus proches qu'on n'aurait pu le penser dans cette partie subrectiligne (tabl. II).

L'espacement le plus courant, qui concerne la moitié des mesures (prises de centre à centre), est de $39-41 \mathrm{~m}$, soit 132 , 135 ou 138,5 pieds, valeurs un peu supérieures aux 120 pieds $(35,48 \mathrm{~m}$ ) préconisés par Vitruve (I)e Architectura, VIII, VI, 3). C'est d'ailleurs une distance de cet ordre $(41,11 \mathrm{~m})$ qui a été observée sur le territoire de Vers (Fabre ot al. dir., 2000, p. 80, $\left.\mathrm{n}^{\circ} \mathrm{5} 0\right)$. Une sculc distance est ici un peu inférieure $(37 \mathrm{~m})$, trois autres sont légèrement supérieures $(43-46 \mathrm{~m})$. Seule la distance entre les puits 4 et 5 est nettement plus importante $(77 \mathrm{~m})$, sans atteindre toutefois les chiffres avancés par Dombre.

66. Voir Fabre et al. dir., 2000), p. 68-118, n"w 44, 50) (2), 117 (5), 137, 164 , 168,170 et 171 .

67. Voir Fabre et al. dir., 2000, p. 68-118, n"5 5, 7, 51, 107 el 118.

68. Voir Fabre et al. dir., 2000, p. 68-118, n\% 135-137 et 174.

69. Aux extrémités du Pont du Gard mais aussi du ponceau de Coste Belle à Yers (ibid., n" 47). Sur la commune de Marguerittes, quatre regards, dont un détruit, sont également portés sur le plan de Dombre $(1 / 10000,1844)$ dont il a été question plus haut (Fabre et al. dir., 2000, p. $\left.103, \mathrm{n}^{\circ} 135-138\right)$.

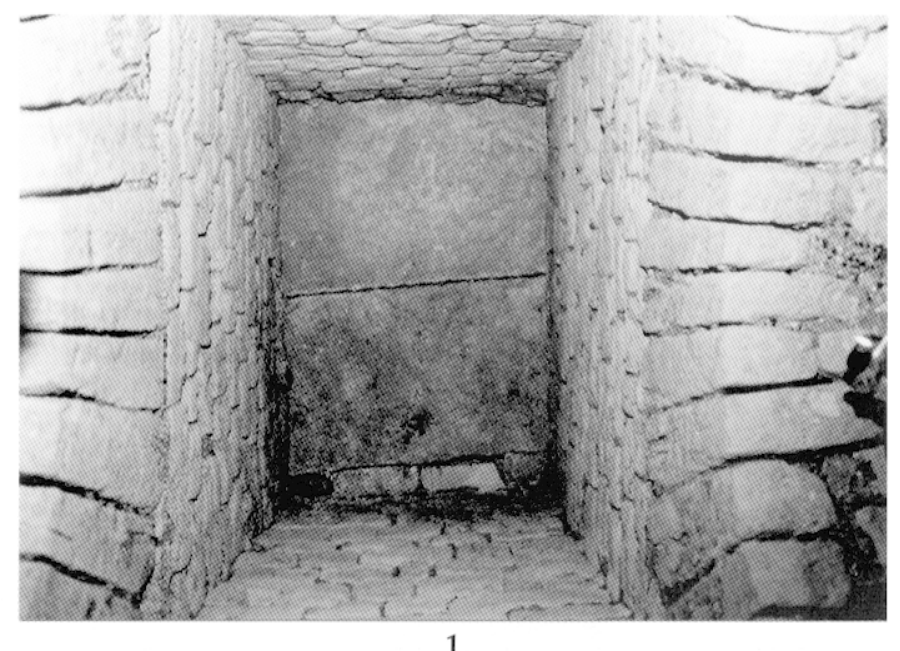

1

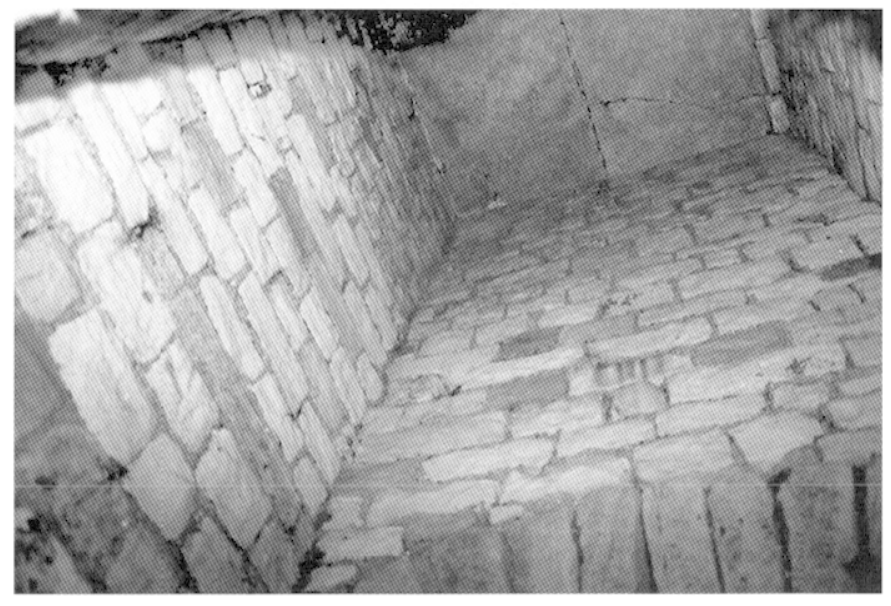

2

Fig. 74 - Regards de Font en Gour : 1, deuxième regard à $84 \mathrm{~m}$ au sud du sile; 2 , sixième regard à $279 \mathrm{~m}$ au sud du site; les moellons sont en calcaire gris-beige alors que les claveaux sont taillés dans la molasse calcaive langhienne (clichés GEO-TER).

Ces dix regards présentent une section rectangulaire uniforme $(1,32 \mathrm{~m}$ x $0,90 \mathrm{~m}$ à la base). Celui du Mas de Gleyze est de même gabarit avec une longueur qui tend à se réduire $(1,25 \mathrm{~m})$ dans la partie haute. En revanche, celui du Mas de Pazac est plus large $(1,15 \mathrm{~m})$. Il semble cependant qu'une largeur de $0,90 \mathrm{~m}$ soit courante. En effet, on la retrouve dans deux des cinq puits du village de Sernhac où les trois autres présentent une largeur de 1,10 m (Fabre et al. dir., 2000), p. 99,

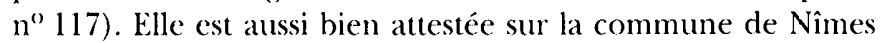
(ibid., p. 110-114, n's 164, 170, 171).

Ces dix puits, et celui qui a été exploré au Mas de Gleyre, ont conservé une couverture de deux ou trois dalles en calcaire gréseux, invisibles en surface. Au Mas de Gleyze, où l'une des dalles a été retaillée à l'époque moderne pour pratiquer un accès, on a pu observer que cette couverture avait une épaisseur de $22 \mathrm{~cm}$ et se situait à moins de $20 \mathrm{~cm}$ sous la surface. Il est très probable que ces dalles sont antiques et marquent le sommet du regard. Celui-ci présente, dans tous les cas, deux types d’appareil superposés : à la base et sur la plus grande 
Tabl. II - Aqueduc de vîmes: espacement des regards à l'aual de Font en Gour:

\begin{tabular}{|c|c|c|}
\hline$N^{\circ}$ du puits & $\begin{array}{c}\text { Distance par rapport } \\
\text { à I'entrée }(\text { en } \mathbf{m})\end{array}$ & $\begin{array}{c}\text { Espacement avec le puits } \\
\text { précédent en } \mathbf{m} \text { et (en pieds romains) }\end{array}$ \\
\hline 1 & 39 & $40(135,27)$ \\
\hline 2 & 84 & $45(152,18)$ \\
\hline 3 & 123 & $39(131,89)$ \\
\hline 4 & 162 & $39(131,89)$ \\
\hline 5 & 239 & $77(260,39)$ \\
\hline 6 & 279 & $40(135,27)$ \\
\hline 7 & 325 & $46(155,56)$ \\
\hline 8 & 362 & $37(125,12)$ \\
\hline 9 & 403 & $41(138,65)$ \\
\hline 10 & 446 & $43(145,41)$ \\
\hline
\end{tabular}

hauteur, un petit appareil de moellons (en calcaire gréseux ou en calcaire gris-beige comme indiqué plus haut) tout à fait comparable à celui qui caractérise les murs du canal; au couronnement, quelques assises en appareil irrégulier ou plus gros, également lié au mortier. Il est peu probable que cette partie haute corresponde à une reprise du bâti dans l'Antiquité ou par la suite. Elle correspond sans doute à la partie du regard qui, à l'origine, n'ćtait pas enterrée. Au Mas de Gleyze, la hauteur de cette partie supérieure du puits est de $0,80 \mathrm{~m}$ alors que celle du petit appareil est de $1,57 \mathrm{~m}$ au-dessus de l'extrados de la voûte.

I a hauteur des regards varie en croissant vers l'aval. Celle du puits $\mathrm{n}^{\circ} \mathrm{l}$ est de $1,35 \mathrm{~m}$ de l'intrados de la voûte à la base de la dalle qui coiffe le puits. Au Mas de Gleyze, elle atteint 2,72 m (fig. 75) pour se situer aux environs de $3 \mathrm{~m}$ au Mas de Pazac. Au-delà de ce point et après un net changement de direction, l'aqueduc est implanté moins profondément, comme on a pu l'observer $1400 \mathrm{~m}$ plus loin aux Cairades où l'intrados se situe à $1,70 \mathrm{~m}$ de la surface (Fabre et al. dir., 2000, p. 102, n 124A) et où l'on a pu mesurer la largeur de sa tranchéc de fondation : $4,20 \mathrm{~m}$ en partic haute et $2,50 \mathrm{~m}$ en partie basse.

\section{LES PENTES}

Entre Sernhac (Le (yrès) et Bezouce (roie ferrée), sur une distance de $5092,30 \mathrm{~m}$, nous disposions de cinq points pour calculer la pente du canal (Fabre $e_{t} a l$. dir., 2000 , pl. X, $n^{\text {os }} 118$, $119,124 \mathrm{~B}$ et 125) qui n'est d'ailleurs pas facile à ćtablir car le radier est généralement ennoyé et couvert de sédiments. Ainsi, au puits de Pazac, son altitude a été déterminée par rapport à un point coté sur la margelle $(70,282 \mathrm{~m})$, à l'aide d'une mire qui, après cnlèrement des sédiments meubles, a permis de mesurer une profondeur de $6,92 \mathrm{~m}$. Dans un premier temps, nous avions considéré que le radier était couvert de concrétions sur $15 \mathrm{~cm}$, comme on a pu le mesurer en plusieurs endroits à l'amont, et retenu la cote de 63,21 m (Fabre et al. dir., 2000, p. 102), mais on ne peut exclure qu'elle approche ou égale 63,36 m si les dépôts au fond ont été moins importants dans ce secteur.

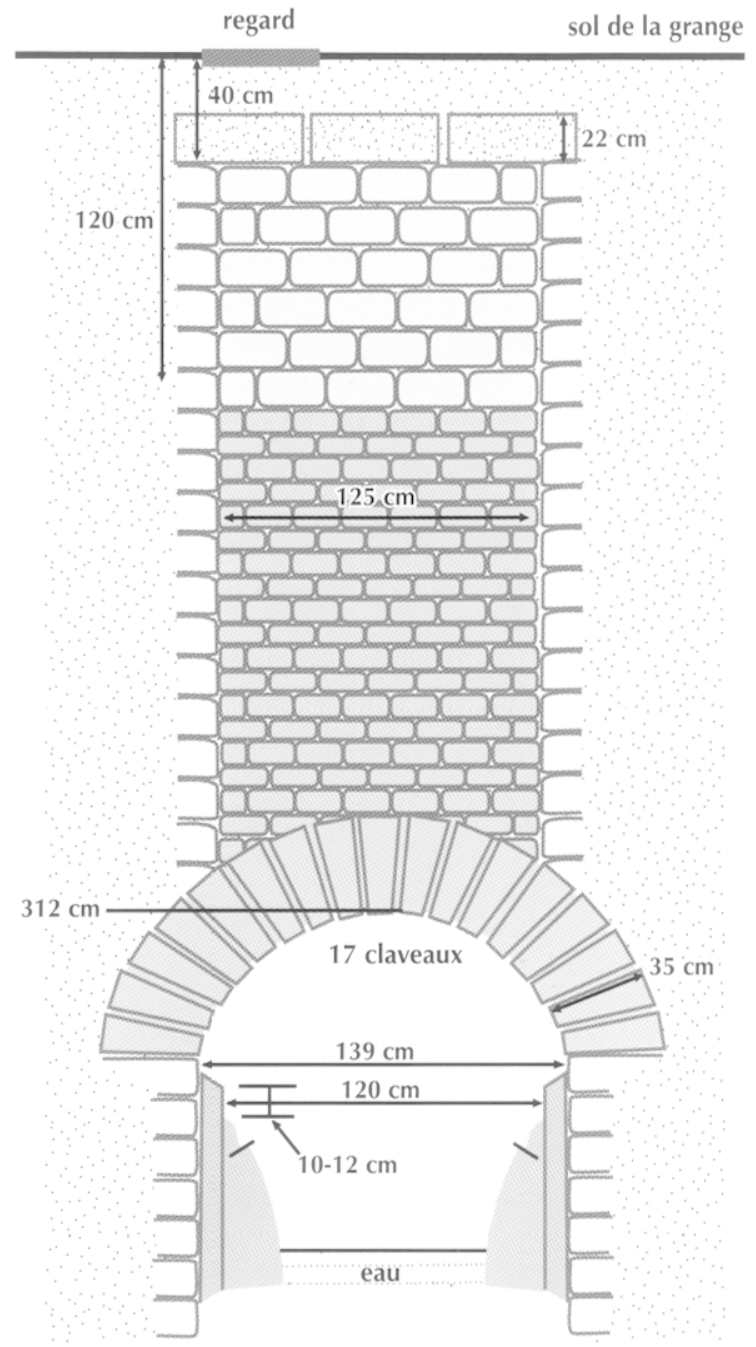

Fig. 75 - Coupe schématique sur le regard du Mas de (ilegze (dessin (r. Fabre, CNRS; DAOD). Carbon, (BLO-TER).

Lors des nouvelles recherches de terrain nous avons vérifié trois de ces points (Le Grès, Font en Gour et Bezouce, voie ferrée) et complété les données au Mas de Gleyze en privilégiant des mesures à l'intrados de la voûte, mais aussi cn cotant le haut du chanfrein du curelage comme nous $r$ invitait une étude récente ${ }^{70}$ (Bossy et al., 2000) (tabl. III).

En supposant que le canal ait une hauteur sous voûte pratiquement constante, le profil établi à partir des mesures de l'intrados montre que cette partie de l'aqueduc présente une pente assez régulière de $14,34 \mathrm{~cm} / \mathrm{km} \pm 1,50$ (fig. 76 ) ; en tout

70. Cette opération a été réalisée le 24 octobre 2000. les points de nivellement ont été faits en deux ctapes: 1) nivellement d'un point caractéristique fixe et réutilisable à l'avenir, en surface et à proximité de laqueduc, en utilisant une antenne (ips (le raccordement absolu par rapport au réseau nivelé de l'IGN. est opéré par l'intermédiaire d'une seconde antenne (iPS implantée sur un repère de nivellement de l'ICX) ; 2) nivellement de différents points caractéristiques du canal de l'aqueduc par rapport au point de nivellement en surface, gràce à une mire et un niveau optique. I a multiplication des mesures en ces points a permis d'obtenir une précision satisfaisante $(1 \mathrm{~cm})$. 
cas, plus faible que celle que nous avions précédemment publiée (Fabre et al. dir., 2000, pl. X: $23 \mathrm{~cm} / \mathrm{km}$ ). En revanche, le profil réalisé à partir des mesures du haut du cuvelage est moins régulier: $35 \mathrm{~cm}$ pour $1411 \mathrm{~m}(24,80 \mathrm{~cm} / \mathrm{km})$ entre I.e Grès et Font en Gour, et $30 \mathrm{~cm}$ pour $3681 \mathrm{~m}(8,15 \mathrm{~cm} / \mathrm{km})$ à l'aval jusqu'à Bezouce. Cette anomalie s'explique, en fait, par une particularité du chanfrein au Grès. Le tableau III montre, en effet, que la hauteur qui sépare le haut du chanfrein de l'intrados y est nettement plus réduite qu'ailleurs où elle se situe entre 54 et $61 \mathrm{~cm}$. On dispose, pour cette section, de relevés effectués par J.-L. Paillet qui a coté le radier à $64,015 \mathrm{~m}$ et la base du chanfrein à 65,244 m (Fabre et al., 1999, p. 217, fig. 4) ; celui-ci avait donc une hauteur de $11 \mathrm{~cm}$. En combinant les mesures de Paillet et les nôtres, on observe, en outre, que la hauteur du canal est ici de $1,78 \mathrm{~m}$ et celle des encroûtements au fond de $15,5 \mathrm{~cm}$.

Nous avons aussi tenté d'évaluer la cote du radier. Pour cela, nous avons tenu compte des hauteurs moyennes du canal sous la voûte $(180 \mathrm{~cm} \pm 3)$ et du cuvelage, sachant que l'on a observé ailleurs une variation de $5 \mathrm{~cm}$ dans la hauteur des murs latéraux (1,30 m à 1,35 m, selon Paillet, 2000, p. 361). En tenant compte des mesures prises sur le terrain et portées sur la figure 73 , on peut avancer des hypothèses argumentées sur l'altitude du radier qui tiennent compte d'une légère variation de la hauteur du canal proposée en fonction des mesures effectuées jusqu'au fond après enlèvement des sédiments meubles (tabl. IV). Cela n'a pas grande influence sur la pente moyenne qui reste faible: la variation de la hauteur du canal qui entraine celle du radier par rapport à celle de l'intrados (3 $\mathrm{cm}$ au plus) n'augmente la pente moyenne que de $1 \mathrm{~cm}(15,4 \mathrm{~cm} / \mathrm{km})$. En revanche, l'encroûtement du fond paraît avoir une épaisseur variable: $15 \mathrm{~cm}$ environ au Grès, en conformité avec ce qui a été observé à l'amont dans les parties où les encroûtements latéraux sont très épais, mais 2 ou $3 \mathrm{~cm}$ seulement à Font en Gour et à Bezouce. Si l'on admet que les $15 \mathrm{~cm}$ du puits du Mas de Gleyze (immergés lors de la mesure) correspondent à des dépôts rejetés du haut, il se pourrait que le canal ait été moins encroûté au fond entre Font en Gour et Bezouce, et ce avant

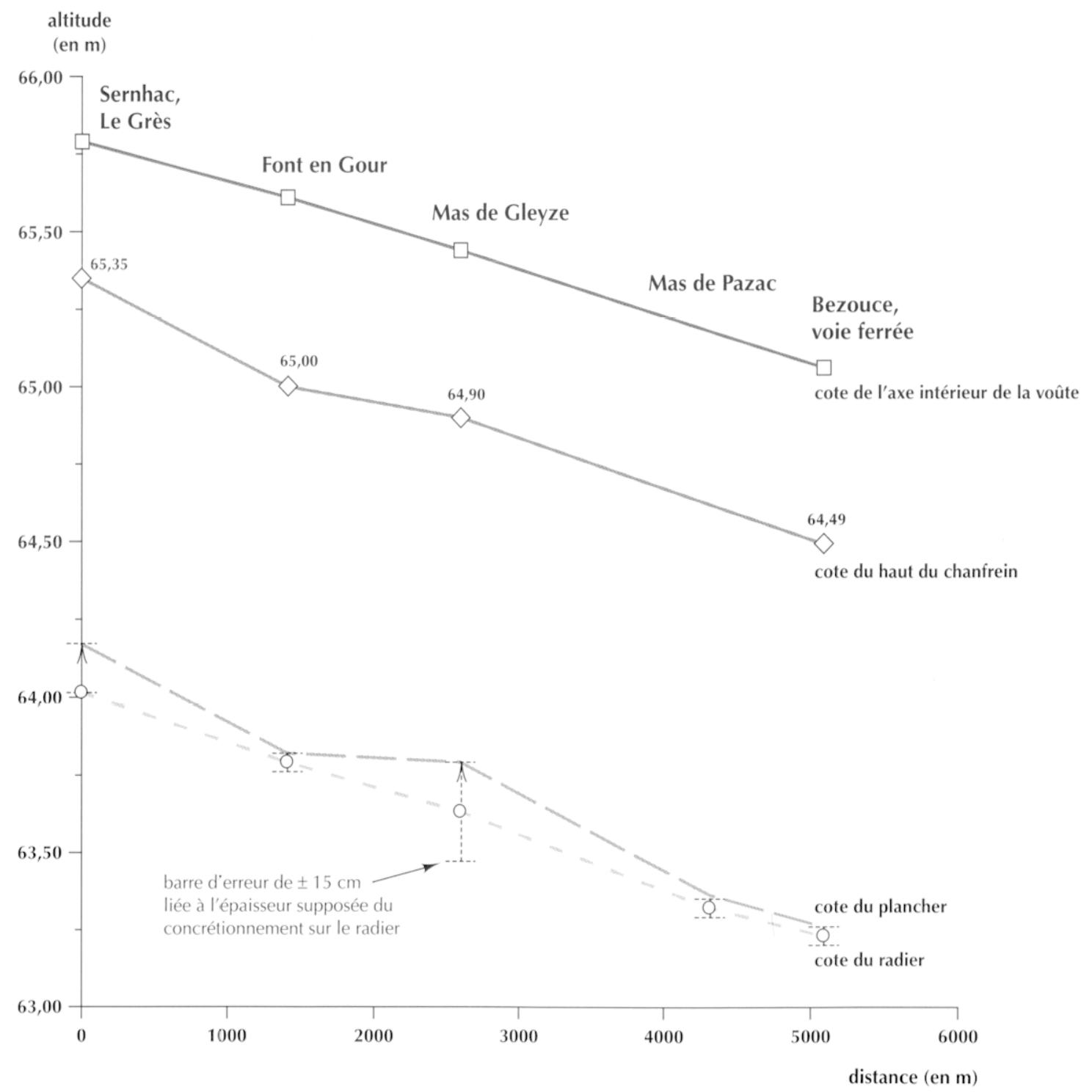

Fig. 76 - Profil du canal entre Sernhac et Bezouce (DAO D. Carbon, (FEO-TER). 
Tabl. III - Aqueduc de Nimes : cotes en mètres relevées sur quatre sections de l'aqueduc.

\begin{tabular}{|l|c|c|c|}
\hline & Intrados & Haut chanfrein & Différence \\
\hline Sernhac, Le Grès & 65,79 & $65,35-65,36$ & 0,435 \\
\hline Lédenon, Font en Gour & 65,61 & $64,99-65,00$ & 0,615 \\
\hline Lédenon, Mas de Gleyze & 65,44 & 64,90 & 0,54 \\
\hline Bezouce, voie ferrée & 65,06 & $64,49-64,46$ & $0,57-0,60$ \\
\hline
\end{tabular}

même d'atteindre la partie où l'on a observé une forte baisse des encroûtements pariétaux.

Si l'on revient au Mas de Pazac (cote sur l'encroûtement de

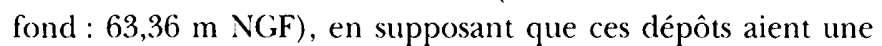
épaisseur de $15 \mathrm{~cm}$, il y aurait une très légère contre-pente jusqu'à Bezouce (1 $720 \mathrm{~m}$ ) alors qu'à l'amont, depuis le Mas de Gleyze $(675 \mathrm{~m})$, la pente serait particulièrement forte $(62 \mathrm{~cm} / \mathrm{km})$, mais pas exceptionnelle (Fabre et al. dir., 2000, p. 135-136). Cette différence demeure d'ailleurs très sensible, même en supposant qu'on ait atteint le radier au Mas de Pazac ; en effet, la pente à l'amont est de $40 \mathrm{~cm} / \mathrm{km}$ environ et à l'aval de $7,5 \mathrm{~cm} / \mathrm{km}$. Un long tronçon à pente moyenne faible peut donc comprendre une partie à forte pente qui, dans ce cas, ne se justifie pas par la topographie. On peut ainsi mieux localiser une de ces parties à forte pente $(52 \mathrm{~cm} \pm 11 / \mathrm{km})$ qui se rencontrent régulièrement entre de longs paliers où la pente est très faible (ibid., p. 137).

\section{FONCTIONNEMENT HYDRAULIQUE ET ENCROÛTEMENTS CARBONATÉS}

Pour expliquer ce phénomène, on peut invoquer une gestion hydraulique originale de stockage et de déstockage qui a été récemment avancée (Bossy et al., 2000, p. 774), mais dont l'étude détaillée reste à faire, en particulier au niveau de l'identification précise de la totalité des biefs, des structures de vannage des seuils et de décharges latérales. Il est possible qu'une structure de régulation ait existé entre le Mas de Gleyze et le Mas de Pazac. En ce point toutefois, un vannage latéral pose problème pour l'évacuation de l'écoulement dans ce contexte de topographie plane où l'aqueduc est profondément enterré; en revanche, un seuil-chute sur le canal pour le stockage peut se concevoir et rendre compte de la forte pente observée dans ce secteur où, par ailleurs, la stratigraphie des cncroûtements pariétaux change très sensiblement.

Près de Bezouce en effet, sous la voie ferréc, on avait observé que l'épaisseur des dépôts carbonatés sur les parois se réduisait à $20 \mathrm{~cm}$ environ, au lieu de $33 \mathrm{~cm}$ à Font en Gour (Fabre, Pey, 1990; Fabre et al. dir., 2000, p. 102, n⿳125; Guendon, Vaudour, 2000, p. 243-244). Malgré une lithostratigraphie comportant toujours un faciès clair rubané suivi d'un faciès tendre et terrigène, des anomalies de croissance affectaient ce dernier (qui n'arrive qu'à la moitié de la hauteur des dépôts antérieurs) et les dernières lamines du faciès clair, elles-mêmes régressives et n'atteignant que les deux tiers de la hauteur des premières lamines (fig. 77). L'origine de cette
Tabl. IV - Aqueduc de Nîmes: propositions pour la hauteur du canal et la cote du radier (au centre, hauteur mesurée dans le canal).

\begin{tabular}{|l|c|c|c|}
\hline & Hauteur canal & Hauteur accessible & Radier \\
\hline Sernhac, Le Grès & 1,78 & 1,62 & 64,015 \\
\hline Lédenon, Font en Gour & 1,82 & 1,79 & 63,79 \\
\hline Lédenon, Mas de Gleyze & 1,81 & 1,65 & 63,63 \\
\hline Bezouce, voie ferrée & 1,83 & 1,80 & 63,23 \\
\hline
\end{tabular}

réduction, qui traduit une baisse nette du niveau de l'eau consécutive à une modification du débit ${ }^{71}$ entre Sernhac et Bezouce, restait cependant très hypothétique : fuite, prélèvement... ?

En réalité, la disposition régressive du concrétionnement interne s'amorce à $460 \mathrm{~m}$ en aval de Font en Gour où les dernières lamines transgressives se trouvent à une dizaine de centimètres sous le sommier de la voûte, alors qu'à l'amont, elles l'atteignent, voire le dépassent légèrement. Au Mas de Gleyze, soit $800 \mathrm{~m}$ plus loin, la géométrie des encroûtements carbonatés sur les piédroits du canal montre une disposition similaire : les encroûtements les plus hauts se situent entre 10 et $12 \mathrm{~cm}$ sous le biseau sommital du cuvelage. Mais ce fait n'est pas comparable en amplitude à celui qui a été observé au Mas de Pazac. Là, en effet, les lamines les plus hautes n'arrivent qu'à $40 \mathrm{~cm}$ environ sous le sommier de la voûte suivant un profil similaire à celui relevé dans le tronçon de la voie ferrée à Bezouce. La régression la plus importante s'opère ainsi nettement entre les Mas de Gleyze et de Pazac, distants de $675 \mathrm{~m}$ environ.

L'origine de cette baisse de niveau ne provient pas d'une évacuation latérale dont la plus proche pourrait être restituée en tête du ruisseau de Bournigue, en amont du regard conservé à la voie ferrée de Sernhac. En effet, la régression des dépôts n'est sensible que beaucoup plus loin, dans une partie où l'on a l'assurance qu'il n'y avait pas de vannage latéral. Il est possible, en revanche, qu'il y ait eu des prélèvements supplémentaires vers la fin du fonctionnement optimal de l'aqueduc pour irriguer des cultures, comme on a pu l'observer sur les ponts à arcades de la Lône, de Pont-Roupt et de Valive (Fabre et al. dir., 2000 , p. 412-413). Il peut aussi exister, comme on l'a déjà évoqué (Fabre, Lerret, 2000, p. 218 et p. 221-222), une relation entre la baisse du niveau de l'eau par fuite dans l'aqueduc au cours de son exploitation antique et une éventuelle déformation superficielle liée à un rejeu de la faille de Nîmes.

Pour dater ce phénomène, on dispose d'une chronologie relative fournie par la stratification des dépôts carbonatés. En demeurant prudent, on pourrait le situer entre le milieu du II $^{\mathrm{e}}$ s. et le milieu du III $^{\mathrm{e}}$ s., peut-être dans la partie centrale de cette période ${ }^{72}$. À priori, il ne s'agit pas de l'un des moments

71. Selon Guendon et Vaudour (2000) la section mouillée a évolué ainsi : au début, $1 \mathrm{~m}^{2}$; lors du dépôt des premières lamines régressives, $0,7 \mathrm{~m}^{2}$; à la fin, lors du dépôt terrigène, $0,5 \mathrm{~m}^{2}$. Avec une vitesse élevée de $1 \mathrm{~m} / \mathrm{s}$, on passerait ainsi de $1 \mathrm{~m}^{3} / \mathrm{s}$ à seulement $500 \mathrm{l} / \mathrm{s}$.

72. Donner une datation absolue n'est pas simple car certains paramètres ne sont pas mesurables, comme la durée de périodes d'arrêt pour travaux ou le rythme des dépôts qui peut rarier. On a cependant proposé 


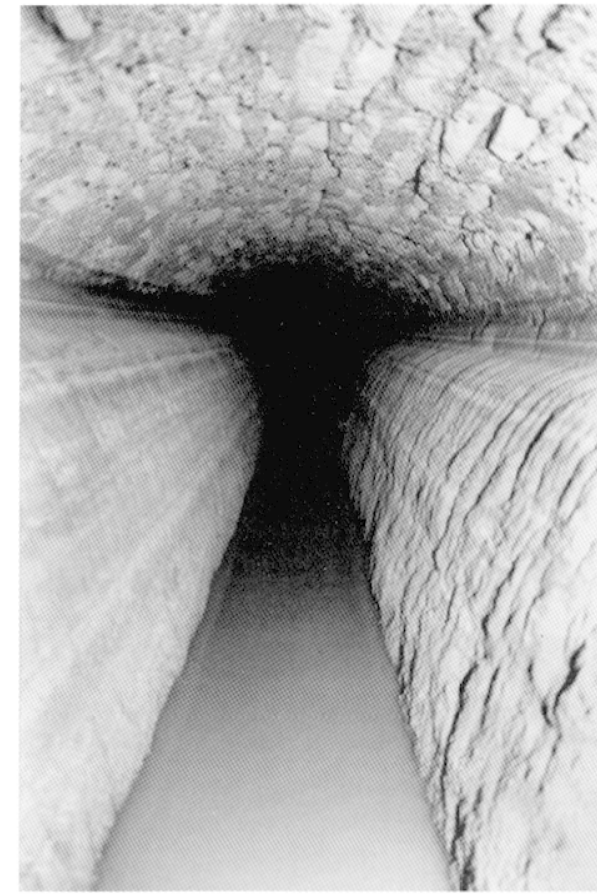

1 - Font en Gour

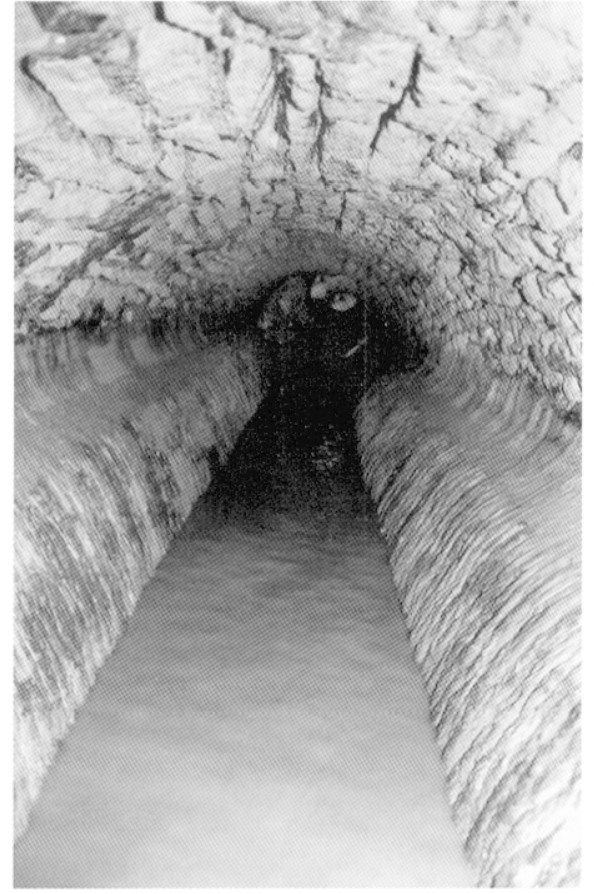

$2-480 \mathrm{~m}$ en aval de Font en Gour

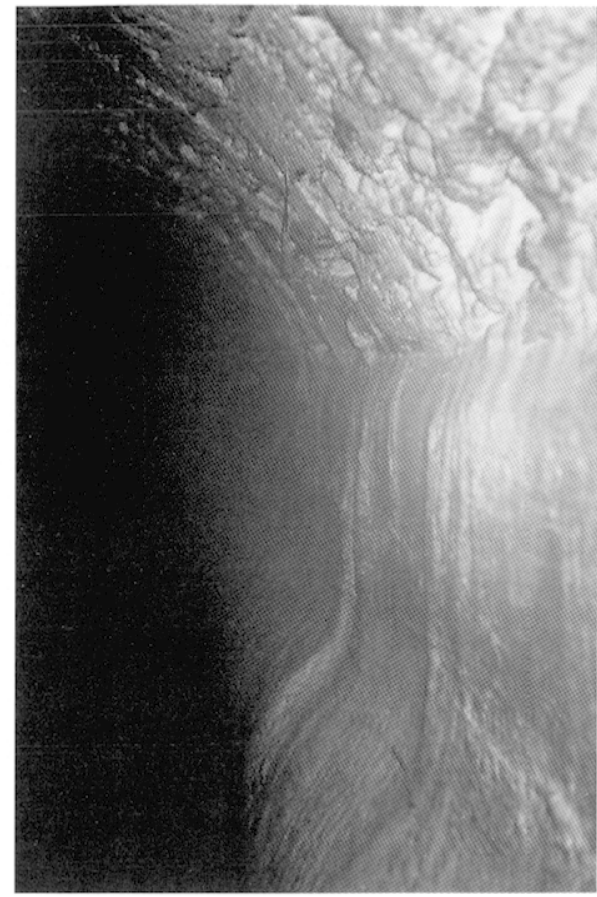

3 - Mas de Pazac (vue subaquatique)

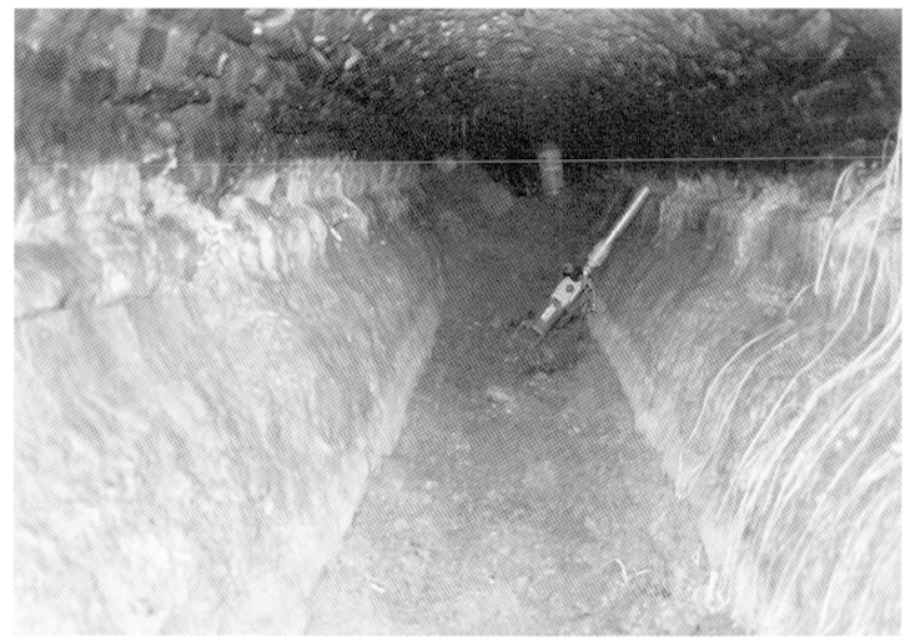

4 - Mas de Gleyze

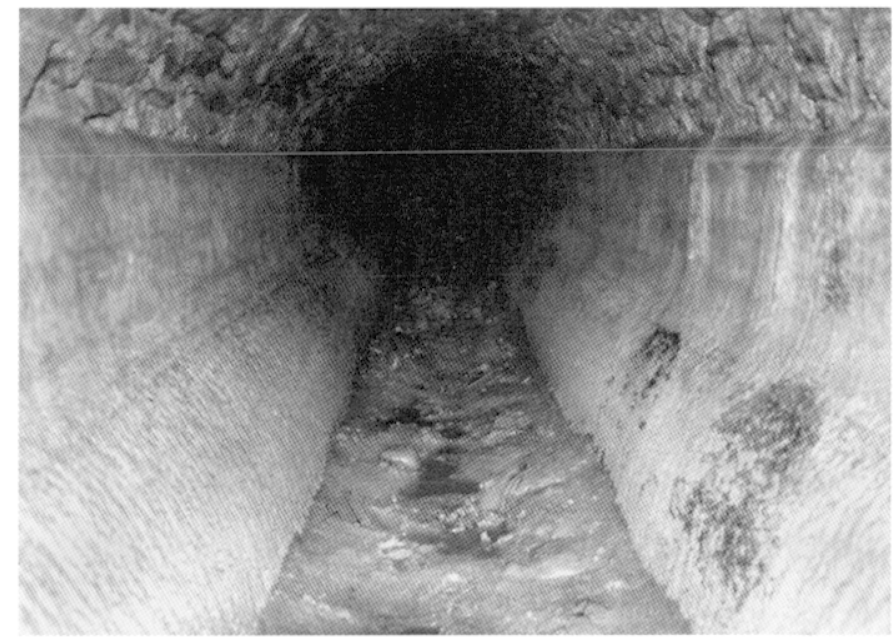

5 - Bezouce, voie ferrée

Fig. 77 - Livolution de la géométrie des encroûtements entre lóont en (Gour al Bezouce (de 1 à 5 ), caractérisée par une régression très netle des dernières lamines de croissance et correspondant à une diminution d'environ $50 \%$ du débit du canal (clichés (FEO)-TER).

où l'on avait supposé une incidence sismique sur l'ouvrage au pont de la Lône. En effet, la destruction partielle du canal sur ce pont, en rapport avec une chute de stalactites sous des arches bouchées, est datée de la première moitié du IV ${ }^{{ }^{\circ}}$ s. ${ }^{73}$ alors

que la pérode de fonctionnement optimal, qui fait suite à une période de réglage de 10 a 20 ans, ait pu durer 140 ans environ et se terminer dans la première moitic du III" s. (Fabre et al. dir., 2000, p. 410-411).

73. Datation obtenue par des céramiques provenant du chantier de reconstruction (Fabre et al. dir., 2000, p. 350)). qu'une première chute de stalactites serait intervenue, en fonction du développement du plancher stalagmitique sur lequel elles se sont brisćes, dans la première moitié du II ${ }^{\circ}$ s. (Combes ot al., 1997, p. 115-118). Il n'est pas exclu cependant, au regard des incertitudes du système de datation, que la première chute de stalactites à la Lône, qu'on arait déja mise en relation avec la construction de contreforts, soit concomitante de la perte importante dans la Vistrenque. Mais l'hypothèse d'une même cause sismique reste fragile. 


\section{ANOMALIES DU BÂTI ET DES ENCROÛTEMENTS}

Deux types d'anomalies, affectant le bâti du canal et les encroûtements carbonatés pariétaux, ont été observés: de longues fractures ouvertes, longitudinales par rapport à l'axe du canal et une déformation souple décalant horizontalement l'ouvrage. Si la seconde ne peut être datée, les premières sont intervenues dans la phase terminale du fonctionnement de l'aqueduc ou après son abandon, puisqu'elles ont affecté les concrétionnements terrigènes les plus récents.

\section{LES FRACTURES OUVERTES}

Le premier type d'anomalies correspond à des fractures longitudinales ouvertes qui affectent les encroûtements carbonatés des piédroits du canal (fig. 78). Déjà observées sous la voie ferrée à Bezouce (Guendon, Vaudour, 2000, p. 243, fig. 138), elles semblaient être la conséquence de vibrations induites par le passage des trains. Mais ces fractures ont désormais été repérées bien au-delà. Elles s'observent généralement dans la partie supérieure des encroûtements et montrent localement un "décollement " du concrétionnement par rapport au cuvelage du canal. C'est surtout le cas au Mas de Gleyze. Aucune fracture n'a pu être mise en évidence dans la partie inférieure des encroûtements ou sur le plancher du canal $\mathrm{cn}$ raison de la présence d'eau et de sédiments, mais leur existence dans ces parties basses n'est pas à exclure.

Les premières fractures ont été vues à $100-110 \mathrm{~m}$ en aval de Font en Gour. Elles s'observent ensuite de manière discontinue jusqu'à la trémie aval, mais avec une augmentation régulière de leur densité, de leur longueur et de leur largeur. Au Mas de Glcyze, ces fissures longitudinales affectent la partie supérieure des encroûtements carbonatés, mais aussi le chanfrein sommital du cuvelage du canal. Certaines fissures présentent un décalage de l'ordre du centimètre qui correspond à un déplacement vers l'intérieur des encroûtements de chaque côté du canal.

Au Mas de Pazac, les encrôtements carbonatés apparaissent fissurés sur une longueur de plus de $30 \mathrm{~m}$ le long de la paroi sud du canal et sur environ $20 \mathrm{~m}$ le long de la paroi opposée. Sur chacune d'elles, la fissure principale affecte la partie supérieure des encroûtements qui s'amincit progressivement vers le haut pour venir biseauter le béton de tuileau. Des fissures secondaires s'obscrvent localement, soit au niveau des encroûtements, soit au niveau des piédroits du canal. L'oricntation des fissures correspond à l'orientation du canal, c'est-à-dire nord-est - sud-ouest. Plusieurs types de fissures ont été observés :

- fissures sans ouverture ou vraisemblablement colmatées, affectant l'encroûtement dans la partic inférieure du bombement et le mur du piédroit ;

- fissures majeures affectant les dépôts carbonatés dans la partie biscautée avec détachement de la plaque encroûtée supérieure ; en plusieurs endroits, la fissure a entraîné un décollement de l'ordre du centimètre de l'encroûtement par rapport au piédroit ;

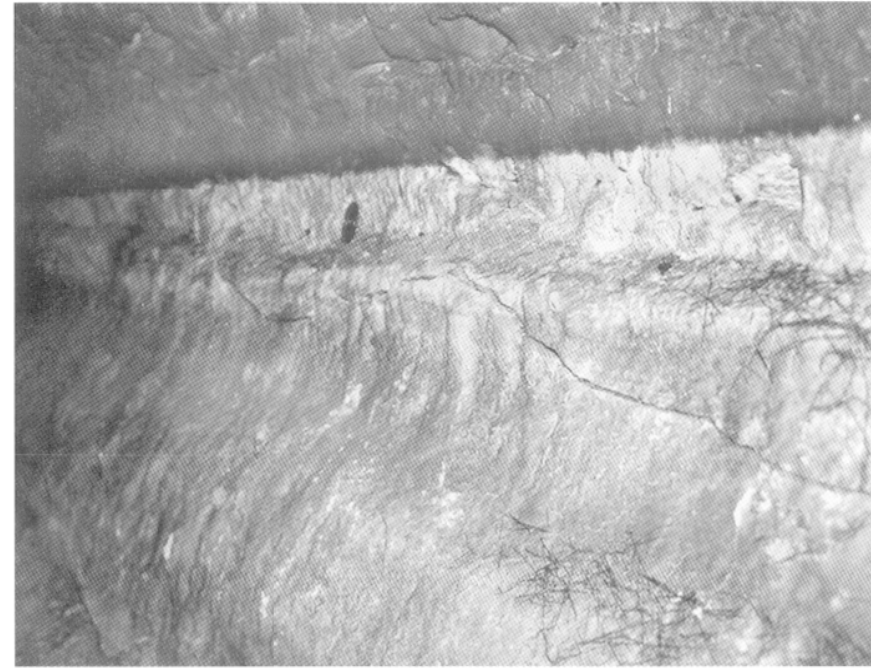

Fig. 78 - Au Mas de Gleyze, les concrétions présentent une géumétrie. régressive; des fractures ouvertes longitudinales affectent les encroûtements et le cuvelage en mortier de tuileau (cliché (GEO)-TER).

- fissures majeures ouvertes (décalage de l'ordre du centimètre) affectant les dépôts carbonatés dans leur partie supérieure.

\section{UNE DÉFORMATION SOUPLE DES ENCROÛTEMENTS}

Une ondulation du canal a étć observée à $32 \mathrm{~m}$ en aral de l'entrée de Font en Gour, sur un tronçon qui ne présente aucun changement de direction au niveau de la voûte (anomalie de structure nord, fig. 70 et 71 ). Elle se manifeste, sur le côté droit du canal, par un renfoncement dans le concrétionnement (alors qu'il est ailleurs parfaitement linćaire sur les tronçons rectilignes de l'aqueduc) et, sur le côté gauche, par un renflement.

\section{HYPOTHÈSES SUR L'ORIGINE DES ANOMALIES OBSERVÉES}

Les fractures ouvertes observées en aval de Font en Gour semblent indiquer que l'aqueduc a subi, dans ce secteur, des déformations suffisamment importantes pour avoir entrainé sa fissuration sur des distances de plusieurs centaines de mètres: dans le secteur de l'étang de Clausonne, les fractures sont de même direction que l'axe du tracé de l'aqueduc (sensiblement nord-sud), alors qu'au Mas de Pazac, cette direction est nord-est - sud-ouest. L'ondulation dont il vient d'être question peut être une autre manifestation de ces déformations.

Bien que ce phénomène s'observe dans la même zone que la perte d'eau, ils n'ont pas la même origine puisque, on l'a vu, l'un est à placer dans l'Antiquité tardive, ou plus tard, alors que l'autre intervient dès le Haut-Empire.

En ce qui concerne le décalage horizontal de l'aqueduc au sud de l'étang de Clausonne, l'absence d'une fracturation visible au niveau des coudes que décrit la structure nous a amenés à 
émettre plusieurs hypothèses. Ce décrochement de l'axe du tracé de l'aqueduc peut être lié à la conception de l'ouvrage si, comme on l'a évoqué plus haut, il s'agissait de passer au-dessous d'un chemin rural. Néanmoins, un tel décalage horizontal du tracé, qui ne conduit pas in fine à un changement de direction, peut avoir une origine tectonique et résulter d'une déformation consécutive au déplacement senestre d'une faille. L'absence de fracture visible dans les encroûtements (qui constituent l'élément le plus rigide de l'ouvrage) pourrait s'expliquer par un comportement général souple (plastique) de cette structure vis-à-vis d'une déformation cisaillante affectant des terrains non consolidés (sables et limons). Néanmoins, des fractures ont pu se créer lors de la déformation, puis être colmatées et fossilisées par les lamines des encroûtements carbonatés qui se sont déposées postérieurement.

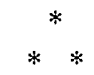

I.e tronçon d'aqueduc qui se développe aux marges de l'étang de Clausonne à partir de Font en Gour justifiait à lui seul une analyse archéologique détaillée. C'est, en effet, la plus longue section intacte actuellement reconnue, qui a permis d'étudier dix regards et d'observer un changement dans les matériaux de construction. Cependant d'autres faits et interrogations justifiaient pleinement l'étude de l'ouvrage dans ce secteur-clé de son tracé, à l'aplomb de la grande faille segmentée de Nîmes et au contact de terrains fortement hétérogènes: d'une part, la régression sur presque $50 \mathrm{~cm}$ de hauteur des encroûtements internes, éléments majeurs dans la compréhension du fonctionnement diachronique du canal ; d'autre part, leur fracturation et des déformations du bâti.

Si l'interprétation des gradients altimétriques parfaitement calés sur le NGF permet de proposer une solution hydraulique de stockage-déstockage modulée de l'écoulement gravitaire, avec vannages, qui pourrait partiellement rendre compte des différents niveaux des concrétionnements internes, il faut souligner sa validité en amont du Grès de Sernhac et les inconnues de l'aval (Bossy et al., 2000); cette hypothèse ne justifie pas, en particulier, la perte d'eau très importante que l'on a pu mieux localiser dans un secteur où l'aqueduc est profondément enterré et qui s'est produite vers la fin du fonctionnement optimal de l'ouvrage.

En France et sur un aqueduc antique, l'analyse de désordres et de certaines anomalies par l'archéosismicité a été utilisée pour la première fois, il y a une dizaine d'années, à l'initiative de l'IRSN avec le CNRS dans le cadre de ses recherches pour l'amélioration de la connaissance de l'aléa sismique (Levret, Combes, 1997). Le cas d'étude de l'aqueduc de Nîmes a permis de poser la problématique dans un cadre pluridisciplinaire et de mettre en place une méthodologie (Fabre, Levret, 2000). Cette démarche a d'abord concerné le pont à arcades de la Lône où s'observent de nombreux désordres: effondrements, fractures, décrochements; en haute Vistrenque, les indices relevés sont d'une grande netteté, mais la difficulté demeure quant à leur rattachement au(x) séisme(s) générateur(s) dûment identifié(s). Il faudrait désormais les confronter à d'autres faits sismiques indubitables, observés dans la région. Malgré ces interrogations, cette contribution apporte des éléments fiables corroborant l'hypothèse de destructions d'origine sismique. Elle invite à poursuivre la recherche sur ce type de monument linéaire de structure rigide-plastique, bon enregistreur des effets sismiques dans la longue durée, en particulier dans des contextes litho-structuraux comparables à ceux que traverse l'aqueduc de Nîmes. 